\title{
Kinetic features of collisionless sheaths around polarized cylindrical emitters from the orbital motion theory
}

\author{
Xin Chen (陈馨), ${ }^{\text {a) }}$ and G. Sanchez-Arriaga ${ }^{\text {b) }}$ \\ Bioengineering and Aerospace Engineering Department, Universidad Carlos III de Madrid, Leganes, \\ Madrid 28911, Spain
}

(Received 24 May 2017; accepted 14 September 2017; published online 2 October 2017)

\begin{abstract}
The kinetic features of the sheath around a cylindrical emitter immersed in collisionless plasma at rest are analysed. After finding self-consistently the electric potential by applying the Orbital Motion Theory to the Vlasov-Poisson system, the local distribution functions are reconstructed and the radial profiles of important macroscopic quantities (plasma densities, currents, and temperatures) are then computed. It is found that there can only be three kinds of holes that are bound by three different boundaries - two related to the constraints from orbital effects and the other due to the electric potential barrier. The results are presented for three regimes: negative probe bias with monotonic and non-monotonic potential and positive probe bias with non-monotonic potential. To understand the variation of macroscopic-quantity radial profiles, three diagrams are presented for kinetic features: the $\epsilon$-diagram for the integration domains of the two orbital invariants, the effective potential, and the local distribution function. The envelope in the $\epsilon l$-diagram is crucial to identify different orbital behaviours, which can be used as a guideline for analytical analyses and serve as one of the criteria to refine the mesh used in numerical calculations. Published by AIP Publishing. https://doi.org/10.1063/1.5004682
\end{abstract}

\section{INTRODUCTION}

The theoretical analysis of the sheath structure around a cylindrical emitter is one of the oldest problems in plasma physics. Part of the major difficulties are related to its kinetic nature. However, the abundant number of applications, such as emissive probes ${ }^{1-3}$ and the charging of dusty-grain ${ }^{4,5}$ and spacecraft, ${ }^{6}$ stimulates theoretical investigations on this topic. The progress on the probe theory can also substantially advance novel applications like low-work-function tethers for deorbiting space debris. ${ }^{7,8}$

A kinetic analysis of collisionless and stationary plasmas involves the solution of the Vlasov-Poisson system. In many scenarios, the symmetries of the problem or other physical features such as the magnetized character of a plasma species typically yield conservation laws. If the number of invariants is sufficient, then a detailed analysis of the particle trajectories can be used to write the densities as a function of the electric potential and, once inserted in Poisson's equation, leads to a single integro-differential equation. This standard methodology has been used in a wide variety of problems, such as plasma in a magnetic cusp, ${ }^{9}$ plasma expansion in a magnetic nozzle, ${ }^{10}$ and astrophysics. ${ }^{11}$ For Langmuir probes, this method is called the Orbital Motion Theory (OMT). The OMT for conventional Langmuir probes was developed in the sixties ${ }^{12-14}$ with some later progresses. ${ }^{15,16}$ Due to the high complexity of the sheath, a self-consistent OMT for emissive probes is theoretically more difficult. With increased emission, the electric field at the emitter is suppressed and a further increase results in a potential minimum, named virtual cathode. ${ }^{17}$ The OMT was partially developed in some asymptotic regimes ${ }^{18,19}$ or

\footnotetext{
a)xin.chen@uc3m.es

b) gonzalo.sanchez@uc3m.es
}

considering radial motion for the emitted electrons and under certain limited conditions for collected species. ${ }^{20}$

In a recent paper, the authors presented a general OMT for emissive probes. $^{21}$ As usual in the OMT, collisions, plasma drift, particle trapping, transient effects, and magnetic fields were ignored. For cylindrical probes immersed in collisionless and stationary plasmas, the conservation of the distribution function, the energy, and the angular momentum was used to formulate the Vlasov-Poisson system by a single integro-differential equation as a function of the radial potential profile. Its numerical solutions were used to identify different EP operational regimes, including orbital-motionlimited (OML)/non-OML current collection and monotonic/ non-monotonic potential, in the parametric domain of probe bias and emission level. The current-voltage characteristics of emissive probes and the floating potential technique were discussed.

The OMT also provides a much deeper insight on the sheath structure. Once the electric potential profile is found, it is then possible to reconstruct the local distribution functions of all the species and, from them, interesting macroscopic quantities like densities, currents, and temperatures can be computed. The goal of this work is to acquire a deep physical understanding of particle orbits and provide detailed results and discussions on the kinetic features.

The work is organized as follows. In Sec. II, the model in Ref. 21 is briefly described as a solid framework of OMT that incorporates all particle species (plasma ions $i$, plasma electrons $e$, and emitted electrons $\mathrm{em}$ ), with orbital motions highlighted as substantive to understand other kinetic features. Three diagrams that illustrate different kinetic features are introduced in Sec. III: (i) $\epsilon$-diagram (the integration domain in terms of two orbital invariants, energy and angular momentum), (ii) $\tilde{u} r$-diagram (effective potential versus radial 
distance to the probe center), and (iii) $\epsilon_{r} \epsilon_{\theta}$-diagram (local distribution functions versus directional-energy plane). In Secs. IV-VI, these diagrams are presented together with the kinetic macroscopic quantities for three operational regimes: negative probe bias with monotonic potential; negative probe bias with non-monotonic potential; and positive probe bias with nonmonotonic potential (operational regimes 6, 5, and 4 in Ref. 21). The conclusions are summarized in Sec. VII.

\section{OMT FOR CYLINDRICAL EMITTERS}

Let us consider a cylindrical emitter-or probe (denoted by subscript $p$ )—with radius $R_{p}$ and bias $\phi_{p}$ relative to a Maxwellian plasma at infinity that has unperturbed density $N_{\infty}$, electron temperature $T_{e}$, and ion temperature $T_{i}$. Electrons are emitted at the probe surface with density $N_{e m p}$ and temperature $T_{e m}$, owing to certain emission mechanisms such as thermionic emission, photoelectric emission, and secondary emission.

Since the axial length of the cylinder is assumed to be much larger than the sheath radius, our analysis ignores potential and distribution function variations along the axial direction. As shown below, radial profiles depend on the following dimensionless parameters:

$$
\beta \equiv \frac{N_{e m p}}{N_{\infty}}, \quad \delta_{i, e m} \equiv \frac{T_{i, e m}}{T_{e}}, \quad \varphi_{p} \equiv \frac{e \phi_{p}}{k_{B} T_{e}}, \quad \rho_{p} \equiv \frac{R_{p}}{\lambda_{D e}},
$$

with subscript $\alpha=i, e, e m$ for three species, $\lambda_{D e}$ $=\sqrt{\varepsilon_{0} k_{B} T_{e} / e^{2} N_{\infty}}$ the plasma electron Debye length, $\varepsilon_{0}$ the vacuum permittivity, and $e$ the elementary charge.

The dimensionless radial coordinate $r$ is defined as the radial distance to the cylinder axis normalized by probe radius $R_{p}$. After defining the $r$-dependent dimensionless electric potential and densities,

$$
\varphi(r) \equiv \frac{e \phi}{k_{B} T_{e}}, \quad n_{i, e}(r) \equiv \frac{N_{i, e}}{N_{\infty}}, \quad n_{e m}(r) \equiv \frac{N_{e m}}{N_{e m p}},
$$

Poisson's equation in cylindrical geometry reads

$$
\begin{gathered}
\frac{1}{r} \frac{\mathrm{d}}{\mathrm{d} r}\left(r \frac{\mathrm{d} \varphi}{\mathrm{d} r}\right)=-\rho_{p}^{2}\left(n_{i}-n_{e}-\beta n_{e m}\right), \\
\varphi(r=1)=\varphi_{p}, \quad \varphi(r \rightarrow \infty) \rightarrow 0 .
\end{gathered}
$$

Based on the OMT, the densities $n_{\alpha}$ on the right-handside of Poisson's equation can be written out explicitly as a function of $\varphi(r)$ according to Vlasov characteristics. ${ }^{21}$ After inserting these densities in Eq. (3), it leads to a single integro-differential equation that can be solved numerically to calculate $\varphi(r)$, without making assumptions about the order of magnitude of the parameters in Eq. (1). Although we have taken single-charged ions $\left(q_{i}=e\right)$ for simplicity, yet it is a hypothesis that can be easily relaxed in the model.

\section{A. Vlasov characteristics}

With the density variation in the axial direction negligible, the normalized particle density $n_{\alpha}$ (of a species $\alpha$ ) at any radial location $r$ can be obtained by integrating the local distribution function $f_{\alpha}$ over the $\left(v_{r}, v_{\theta}\right)$ velocity space,

$$
n_{\alpha}(r)=\iint f_{\alpha}\left(r, v_{r}, v_{\theta}\right) \mathrm{d} v_{r} \mathrm{~d} v_{\theta}
$$

where the normalizations applied are $2 k_{B} T_{\alpha} f_{\alpha} /\left(m_{\alpha} N_{\infty, e m p}\right)$ $\rightarrow f_{\alpha}$ for distribution function, $\sqrt{m_{\alpha} / 2 k_{B} T_{\alpha}} v_{r} \rightarrow v_{r}$ for radial velocity (positive outwards), and $\sqrt{m_{\alpha} / 2 k_{B} T_{\alpha}} v_{\theta} \rightarrow v_{\theta}$ for azimuthal velocity, with $m_{\alpha}$ for particle mass. The $\left(v_{r}, v_{\theta}\right)$ integration domain should contain the velocities of all populations of this species present at $r$.

The Vlasov equation conserves the distribution function along particle orbits. The particle density $n_{\alpha}(r)$ can thus be written as a function of the electric potential $\varphi(r)$. Because plasma species are assumed to be Maxwellian at infinity and emitted electrons are emitted at the probe with a halfMaxwellian (HM) distribution based on the probe temperature $\left(T_{e m}=T_{p}\right)$, the local distribution for the particles existing at $r$ reads

$$
\begin{gathered}
f_{\alpha}\left(r, v_{r}, v_{\theta}\right)=H_{\alpha} \exp \left(-v_{r}^{2}-v_{\theta}^{2}-u_{\alpha r}\right) / \pi, \\
H_{i, e}=1, \quad H_{e m}=2 .
\end{gathered}
$$

The normalized electric potential energy, $u_{\alpha r}(r)$, is defined for each species as

$$
u_{e r}(r) \equiv-\varphi(r), \quad u_{i r}(r) \equiv \frac{\varphi(r)}{\delta_{i}}, \quad u_{e m r}(r) \equiv \frac{\varphi_{p}-\varphi(r)}{\delta_{e m}},
$$

relative to the potential energy at the origin of a species. The term origin refers to infinity for plasma ions/electrons $(r \rightarrow \infty$ for $\alpha=i, e)$ and probe surface for emitted electrons ( $r=1$ for $\alpha=e m$ ). The subscript $r$ is often used in this work to emphasize that such a quantity depends on $r$.

Due to the cylindrical symmetry of the problem and its stationary character, the normalized angular momentum and the normalized transverse total energy,

$$
l_{\alpha} \equiv r v_{\theta}, \quad \epsilon_{\alpha} \equiv v_{r}^{2}+v_{\theta}^{2}+u_{\alpha r},
$$

are conserved along particle orbits. They not only suffice to characterize the orbits but also allow transforming the density integration over the $v_{r} v_{\theta}$-space to the $\epsilon l$-space. After a change of variables, $\left(v_{r}, v_{\theta}\right) \rightarrow\left(\epsilon_{\alpha}, l_{\alpha}\right)$, Eq. (4) becomes

$$
n_{\alpha}(r)=\iint_{2 D_{r}} \frac{f_{\alpha}\left(r, v_{r}, v_{\theta}\right)}{2 \sqrt{l_{\alpha r}^{2}\left(r, \epsilon_{\alpha}\right)-l_{\alpha}^{2}}} \mathrm{~d} \epsilon_{\alpha} \mathrm{d} l_{\alpha}
$$

where $l_{\alpha r}$ is defined as

$$
l_{\alpha r}^{2}\left(r, \epsilon_{\alpha}\right) \equiv l_{\alpha}^{2}+r^{2} v_{r}^{2}=r^{2}\left(\epsilon_{\alpha}-u_{\alpha r}\right) .
$$

The domain of integration $\left(2 D_{r}\right)$ should contain all values of the orbital invariants $\left(\epsilon_{\alpha}, l_{\alpha}\right)$ for the particles present at $r$, which will be discussed in Sec. II B. The factor 2 stands for considering only positive $l_{\alpha}$ and $l_{\alpha r}$ hereafter.

\section{B. Particle orbits}

As usual in the OMT, this work neglects trapped particles. Therefore, a plasma particle $(\alpha=e, i)$ is present at $r$ if its characteristic has the origin at infinity. The plasma 
particles at $r$ are thus composed only of two populations, with the $\epsilon$-domain partitioned as $D_{r}=D_{1 r}+D_{2 r}$. The population characterized by $D_{1} r$ arrives at $r$ for the first time, as incoming particles $\left(v_{r}<0\right)$. Some of these particles continue moving inwards and will be captured by the probe - characterized by a domain, say $D_{0}$. The rest of the particles, $D_{2 r}=D_{1 r}-D_{0}$, are reflected back to $r$ and form as a reflected population that arrives at $r$ for the second time, as outgoing particles $\left(v_{r}>0\right)$. Due to the axial-symmetric character of the electric field, a reflected plasma particle will describe an orbit that is symmetric to the path before reflection, thus heading back to infinity. All these arguments also hold for the emitted electrons $(\alpha=e m)$, by substituting the words infinity, incoming, and $v_{r}<0$, with probe, outgoing, and $v_{r}>0$, respectively. The $D_{1 r}, D_{0}$, and $D_{2 r}$ domains for emitted electrons $(\alpha=e m)$ characterize the particles that reach $r$ for the first time with $v_{r}>0$, that reach infinity, and that are reflected back to the probe and arrive at $r$ again with $v_{r}<0$, respectively. The integration domain $D_{r}$ in Eq. (8) for any species thus reads

$$
D_{r}=D_{1 r}+D_{2 r}=2 D_{1 r}-D_{0} .
$$

The $\epsilon l$-domains at a given $r$ can be presented in a $\epsilon_{\alpha}$-versus$l_{\alpha}^{2}$ plane as shown schematically in Fig. 1 . We here thus look for the boundaries of these domains: the $l_{\alpha r}, l_{\alpha r}^{*}$, and $l_{\alpha}^{*}$ boundary for the non-forbidden, sufficient, and captured condition, respectively.

\section{Non-forbidden condition and $I_{\alpha r}$ boundary}

The particles are forbidden at $r$ if $v_{r}^{2}>0$ cannot be satisfied in Eq. (9), yielding the non-forbidden domain as

$$
D_{N F r}=\left\{\left(\epsilon_{\alpha}, l_{\alpha}\right): \epsilon_{\alpha} \geq u_{\alpha r}(r), l_{\alpha} \leq l_{\alpha r}\left(r, \epsilon_{\alpha}\right)\right\} .
$$

First of all, the condition $\epsilon_{\alpha} \geq u_{\alpha r}$ asks for enough energy to overcome the electric potential barrier at $r$ [see also Eq. (7)]. Due to orbital effects, there is also the orbital barrier at $r$ that forbids the particles with an angular momentum beyond a certain limit. For an energy $\epsilon_{\alpha} \geq u_{\alpha r}$, the orbit with angular momentum $l_{\alpha}^{2}=l_{\alpha r}^{2}\left(r, \epsilon_{\alpha}\right)=r^{2}\left(\epsilon_{\alpha}-u_{\alpha r}\right)$ has $v_{r}=\mathrm{d} v_{\theta} / \mathrm{d} t=0$ at $r$, thus being reflected there. Any higher angular momentum $\left(l_{\alpha}>l_{\alpha r}\right)$ results in $v_{r}^{2}<0$ as more kinetic energy is

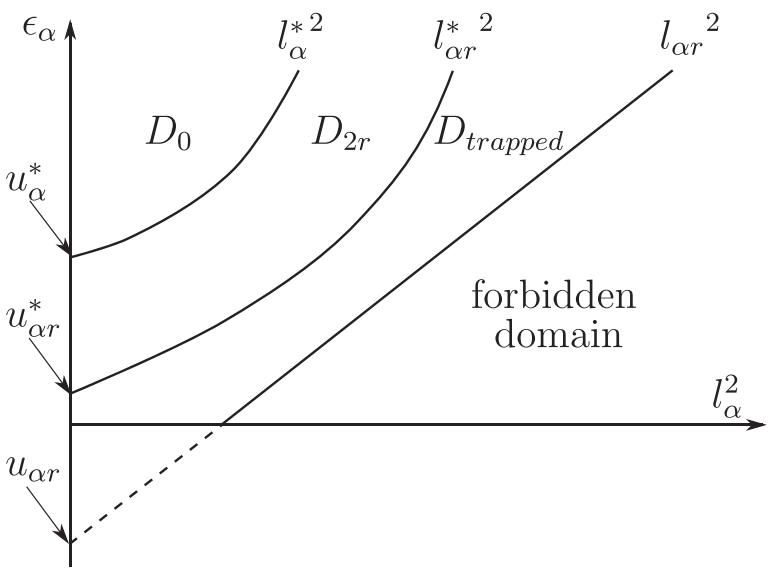

FIG. 1. Schematics of the $\epsilon$-domains in a $\left(\epsilon_{\alpha}, l_{\alpha}^{2}\right)$ plane for a given $r$. The first-arriving domain is $D_{1 r}=D_{0}+D_{2 r}$, and the full integration domain is $D_{r}=D_{1 r}+D_{2 r}$. ascribed to $v_{\theta}$, thus being forbidden at $r$. For instance, for $\epsilon_{\alpha}=u_{\alpha r}$, only a radial orbit $l_{\alpha}=0$ is allowed at $r$. Most importantly, the angular-momentum limit $l_{\alpha r}^{2}$ depends on the energy $\epsilon_{\alpha}$ and appears in Fig. 1 as a straight line. ${ }^{14,15}$ Eventually, the non-forbidden domain corresponds to the region to the left of the $l_{\alpha r}^{2}$ line. This line intersects with the $\epsilon_{\alpha}$-axis at $\epsilon_{\alpha}=u_{\alpha r}$. If $u_{\alpha r}$ is negative, the non-forbidden domain enters the fourth quadrant. Nevertheless, since all particles are released with zero potential energy $\left(u_{\alpha r} \rightarrow 0\right)$ at their origins [see Eq. (6)], the total energy of any particlebeing an orbital invariant and given by Eq. (7) — cannot be negative $\left(\epsilon_{\alpha} \geq 0\right)$. As a consequence, the conditions $l_{\alpha}^{2} \geq 0$ and $\epsilon_{\alpha} \geq 0$ tell that the relevant region for our problem is only the first quadrant in Fig. 1.

\section{Sufficient condition and $I_{\alpha r}^{*}$ boundary}

For a particle to arrive at $r$ for the first time, the non-forbidden condition should be satisfied along the orbit at every $r^{\prime}$ prior to $r$, thus yielding the $D_{1 r}$ domain as

$$
\begin{gathered}
D_{1 r}=\left\{\left(\epsilon_{\alpha}, l_{\alpha}\right): \epsilon_{\alpha} \geq u_{\alpha r}^{*}(r), l_{\alpha} \leq l_{\alpha r}^{*}\left(r, \epsilon_{\alpha}\right)\right\}, \\
u_{\alpha r}^{*}(r) \equiv \max _{r^{\prime} \in S_{r}} u_{\alpha}\left(r^{\prime}\right), l_{\alpha r}^{*}\left(r, \epsilon_{\alpha}\right) \equiv \min _{r^{\prime} \in S_{r}} l_{\alpha r}\left(r^{\prime}, \epsilon_{\alpha}\right), \\
S_{r}=\left\{1 \leq r^{\prime} \leq r \text { for } \alpha=e m, r \leq r^{\prime}<\infty \text { for } \alpha=i, e\right\} .
\end{gathered}
$$

The energy has to be large enough to overcome the highest electric potential hill on the way, while orbital barriers require the angular momentum below the smallest limit. As a result, the $l_{\alpha r}^{*}$ boundary can only coincide or lie to the left of the $l_{\alpha r}$ boundary, which can thus be non-linear to $\epsilon_{\alpha}$ for some energies. Note that $u_{\alpha r}^{*}$ cannot be negative (not as $u_{\alpha r}$ ), due to $u_{\alpha r} \rightarrow 0$ at particle origins.

\section{Captured condition and $I_{\alpha}^{*}$ boundary}

Among the non-forbidden particles, some plasma particles (emitted electrons) can be captured by the probe (infinity), yielding the $D_{0}$ domain as

$$
\begin{gathered}
D_{0}=\left\{\left(\epsilon_{\alpha}, l_{\alpha}\right): \epsilon_{\alpha} \geq u_{\alpha}^{*}, l_{\alpha} \leq l_{\alpha}^{*}\left(\epsilon_{\alpha}\right)\right\}, \\
u_{\alpha}^{*} \equiv \max _{r^{\prime} \in S} u_{\alpha}\left(r^{\prime}\right), \quad l_{\alpha}^{*}\left(\epsilon_{\alpha}\right) \equiv \min _{r^{\prime} \in S} l_{\alpha r}\left(r^{\prime}, \epsilon_{\alpha}\right), \\
S=\left\{1 \leq r^{\prime}<\infty\right\} .
\end{gathered}
$$

Such an $l_{\alpha}^{*}$ boundary, which contains the highest electric potential hill and the smallest angular momentum limit for all $r$ locations, is invariant with $r$. The $l_{\alpha}^{*}$ boundary, which coincides or lies to the left of the $l_{\alpha r}^{*}$ boundary, can also be non-linear to $\epsilon_{\alpha}$ for some energies.

With both the sufficient domain $D_{1 r}$ and the captured domain $D_{0}$ identified, the total integration domain $D_{r}$ in Eq. (8) can thus be found by using Eq. (10). At the end, to determine $D_{r}$, the key is thus to find the $l_{\alpha r}^{*}$ and $l_{\alpha}^{*}$ boundaries (together with the energy preconditions implied) by examining the variation of the non-forbidden $l_{\alpha r}^{2}$ line with $r$ [see Sec. III A].

\section{Trapped orbits}

Although this work neglects the particles that could be trapped due to transient or collisional effects, we now discuss 
their $\epsilon l$-domain for completeness. Trapped orbits should satisfy the non-forbidden condition and, additionally, do not connect with the particle origin (a condition that defines the $D_{1 r}$ domain). Therefore, the trapped-orbit domain at $r$ is

$$
D_{\text {trapped }}=D_{N F r}-D_{1 r} \text {. }
$$

Although our analysis assumes no trapped particles, such a model could be extended if a law for the distribution function inside this trapped domain is prescribed.

\section{Macroscopic Quantities}

After substituting into Eq. (8) the $D_{r}$ domain given by Eqs. (10), (12), and (13), the particle density reads

$$
\begin{aligned}
n_{\alpha}(r)= & \frac{H_{\alpha}}{\pi} \iint_{2 D_{1 r}-D_{0}} \frac{\exp \left(-\epsilon_{\alpha}\right) \mathrm{d} \epsilon_{\alpha} \mathrm{d} l_{\alpha}}{\sqrt{l_{\alpha r}^{2}\left(r, \epsilon_{\alpha}\right)-l_{\alpha}^{2}}} \\
= & \frac{2 H_{\alpha}}{\pi} \int_{u_{\alpha r}^{*}(r)}^{\infty} \exp \left(-\epsilon_{\alpha}\right) \arcsin \frac{l_{\alpha r}^{*}\left(r, \epsilon_{\alpha}\right)}{l_{\alpha r}\left(r, \epsilon_{\alpha}\right)} \mathrm{d} \epsilon_{\alpha} \\
& -\frac{H_{\alpha}}{\pi} \int_{u_{\alpha}^{*}}^{\infty} \exp \left(-\epsilon_{\alpha}\right) \arcsin \frac{l_{\alpha}^{*}\left(\epsilon_{\alpha}\right)}{l_{\alpha r}\left(r, \epsilon_{\alpha}\right)} \mathrm{d} \epsilon_{\alpha} .
\end{aligned}
$$

Such an integration does not only depend on the electric potential $\varphi$ at $r$ but also that at other $r$ locations (see the star signs). Using Eq. (15) in Eq. (3) leads to an integro-differential equation for the Vlasov-Poisson system, which can be solved numerically with an iterative scheme to compute $\varphi(r)$.

Once $\varphi(r)$ is found, other macroscopic quantities can be calculated. With the distribution average of a quantity $g\left(r, v_{r}, v_{\theta}\right)$ defined as

$$
\left\langle g\left(r, v_{r}, v_{\theta}\right)\right\rangle=\frac{\int g\left(r, v_{r}, v_{\theta}\right) f\left(r, v_{r}, v_{\theta}\right) \mathrm{d} v_{r} \mathrm{~d} v_{\theta}}{n(r)},
$$

the radial and azimuthal average velocities can be written as

$$
\begin{aligned}
\left\langle v_{r}\right\rangle & = \pm \frac{H_{\alpha}}{\pi r n_{\alpha}} \iint_{D_{0}} \exp \left(-\epsilon_{\alpha}\right) \mathrm{d} \epsilon_{\alpha} \mathrm{d} l_{\alpha} \\
& = \pm \frac{H_{\alpha}}{\pi r n_{\alpha}} \int_{u_{\alpha}^{*}}^{\infty} l_{\alpha}^{*}\left(\epsilon_{\alpha}\right) \exp \left(-\epsilon_{\alpha}\right) \mathrm{d} \epsilon_{\alpha}, \\
\left\langle v_{\theta}\right\rangle & =0 .
\end{aligned}
$$

where the $-(+)$ sign corresponds to plasma species (emitted electrons). The $\left\langle v_{r}\right\rangle$ integration involves only $D_{0}$ because the orbits characterized by $D_{2 r}$ appear at $r$ twice, yet with opposite radial velocity. The moment $\left\langle v_{r}\right\rangle$ is very useful because it is related to the current density per unit length as $I_{\alpha}=2 \pi R_{p} q_{\alpha} N_{\infty, e m p} \sqrt{2 k_{B} T_{\alpha} / m_{\alpha}} \times r n_{\alpha}\left\langle v_{r}\right\rangle$. After normalizing with the electron thermal random current $I_{t h}$ $=2 \pi R_{p} e N_{\infty} \sqrt{k_{B} T_{e} / 2 \pi m_{e}}$, one finds

$$
\begin{aligned}
i_{\alpha} & \equiv \frac{I_{\alpha}}{I_{t h}}=\frac{2 G_{\alpha}}{\sqrt{\pi}} \int_{u_{\alpha}^{*}}^{\infty} l_{\alpha}^{*}\left(\epsilon_{\alpha}\right) \exp \left(-\epsilon_{\alpha}\right) \mathrm{d} \epsilon_{\alpha}, \\
G_{i} & =-\sqrt{\delta_{i} / \mu_{i}}, \quad G_{e}=1, \quad G_{e m}=-2 \beta \sqrt{\delta_{p}},
\end{aligned}
$$

being invariant with $r$ and positive for collected electron current. The mass ratio $\mu_{i} \equiv m_{i} / m_{e}$ appears in this current calculation but not in the Vlasov-Poisson system.
Additional interesting quantities are kinetic temperatures. They are given by the second central moments with respect to the average velocity as

$$
\begin{aligned}
& \tilde{T}_{\alpha r}=\left\langle\left(v_{r}-\left\langle v_{r}\right\rangle\right)^{2}\right\rangle=\left\langle v_{r}^{2}\right\rangle-\left\langle v_{r}\right\rangle^{2}, \\
& \tilde{T}_{\alpha \theta}=\left\langle v_{\theta}^{2}\right\rangle,
\end{aligned}
$$

where they are normalized to $T_{\alpha}$ of each species and $\left\langle v_{r}\right\rangle$ is given by Eq. (18). For convenience, we use Eq. (7) to write the other two terms (average kinetic energies) as

$$
\begin{aligned}
& \left\langle v_{r}^{2}\right\rangle=\left(\left\langle\epsilon_{\alpha}\right\rangle-u_{\alpha r}\right) / 2+K, \\
& \left\langle v_{\theta}^{2}\right\rangle=\left(\left\langle\epsilon_{\alpha}\right\rangle-u_{\alpha r}\right) / 2-K
\end{aligned}
$$

with

$$
\begin{aligned}
\left\langle\epsilon_{\alpha}\right\rangle= & \frac{H_{\alpha}}{\pi n_{\alpha}} \iint_{2 D_{1 r}-D_{0}} \frac{\epsilon_{\alpha} \exp \left(-\epsilon_{\alpha}\right) \mathrm{d} \epsilon_{\alpha} \mathrm{d} l_{\alpha}}{\sqrt{l_{\alpha r}^{2}\left(r, \epsilon_{\alpha}\right)-l_{\alpha}^{2}}} \\
= & \frac{2 H_{\alpha}}{\pi n_{\alpha}} \int_{u_{\alpha r}^{*}(r)}^{\infty} \epsilon_{\alpha} \exp \left(-\epsilon_{\alpha}\right) \arcsin \frac{l_{\alpha r}^{*}\left(r, \epsilon_{\alpha}\right)}{l_{\alpha r}\left(r, \epsilon_{\alpha}\right)} \mathrm{d} \epsilon_{\alpha} \\
& -\frac{H_{\alpha}}{\pi n_{\alpha}} \int_{u_{\alpha}^{*}}^{\infty} \epsilon_{\alpha} \exp \left(-\epsilon_{\alpha}\right) \arcsin \frac{l_{\alpha}^{*}\left(\epsilon_{\alpha}\right)}{l_{\alpha r}\left(r, \epsilon_{\alpha}\right)} \mathrm{d} \epsilon_{\alpha}, \\
K= & \frac{H_{\alpha}}{\pi r^{2} n_{\alpha}} \int_{u_{\alpha r}^{*}(r)}^{\infty} \exp \left(-\epsilon_{\alpha}\right) l_{\alpha r}^{*} \sqrt{l_{\alpha r}^{2}-l_{\alpha r}^{*} 2} \mathrm{~d} \epsilon_{\alpha} \\
& -\frac{H_{\alpha}}{2 \pi r^{2} n_{\alpha}} \int_{u_{\alpha}^{*}}^{\infty} \exp \left(-\epsilon_{\alpha}\right) l_{\alpha}^{*} \sqrt{l_{\alpha r}^{2}-l_{\alpha}^{*} 2} \mathrm{~d} \epsilon_{\alpha} .
\end{aligned}
$$

The term $\left\langle\epsilon_{\alpha}\right\rangle$ represents the average total energy and, as shown by Eqs. (22) and (23), $K$ indicates the kinetic energy exchange between the radial and azimuthal directions.

The general procedure to compute macroscopic quantities involves first solving Eq. (3) for $\varphi(r)$ and then evaluating the integrals for $n_{\alpha}$ [which has been used in Eq. (3)], $i_{\alpha}$, $\tilde{T}_{\alpha r}$, and $\tilde{T}_{\alpha \theta}$. However, for some special cases, the macroscopic quantities can be evaluated at the probe (denoted by the subscript $R$, instead of $p$ for conditions set at the probe) beforehand with no need of solving Poisson's equation. For instance, for emitted electrons with negative probe bias and monotonic potential, the HM distribution at the probe simply gives $n_{e m R}=1, i_{e m}=G_{e m}, \tilde{T}_{e m \theta, R}=1 / 2$, and $\tilde{T}_{e m r, R}=1 / 2$ $-1 / \pi$. For a repelled plasma species $\left(\alpha=i, e\right.$ for $\varphi_{p}>0$ and $\varphi_{p}<0$, respectively) with monotonic potential, due to $u_{\alpha r}^{*}$ $=u_{\alpha r}$ and $l_{\alpha r}^{*}=l_{\alpha r}$, one finds $n_{\alpha R}=\exp \left(-u_{\alpha R}\right) / 2, i_{\alpha}$ $=G_{\alpha} \exp \left(-u_{\alpha R}\right), \tilde{T}_{\alpha \theta, R}=1 / 2$, and $\tilde{T}_{\alpha r, R}=1 / 2-1 / \pi$.

Another interesting case that can be handled beforehand is the OML condition for attracted plasma species $(\alpha=i, e$ for $\varphi_{p}<0$ and $\varphi_{p}>0$, respectively). Under OML conditions, whether an attracted particle can be captured by probe is governed by the non-forbidden condition at probe only $\left(l_{\alpha}^{*}=l_{\alpha R}\right.$ for all $\left.\epsilon_{\alpha} \geq u_{\alpha}^{*}=0\right)$. In this case, the macroscopic quantities are $n_{\alpha R}=1 / 2, i_{\alpha}=G_{\alpha}\left[\operatorname{erfcx}\left(\sqrt{-u_{\alpha R}}\right)\right.$ $\left.+2 \sqrt{-u_{\alpha R} / \pi}\right], \tilde{T}_{\alpha \theta R}=\left(1-u_{\alpha R}\right) / 2$, and $\tilde{T}_{\alpha r, R}=\left(1-u_{\alpha R}\right) /$ $2-\left[\sqrt{\pi} \operatorname{erfcx}\left(\sqrt{-u_{\alpha R}}\right)+2 \sqrt{-u_{\alpha R}}\right]^{2} / \pi^{2}$.

\section{KINETIC FEATURES}

In order to discuss the kinetic features of an emissive sheath, it is necessary to construct the local distribution 
function at any given $r$, of which the integrations give the macroscopic quantities. As we will see, a representation of this distribution versus the $\epsilon_{r} \epsilon_{\theta}$-plane (the directional energies) is better than the usual $v_{r} v_{\theta}$-plane. The local distribution functions, for instance, see Fig. 4(c), exhibit different holes for the regions that are unpopulated by particles. The variation of these holes with $r$ can be explained by looking at the already mentioned $\epsilon l$-diagram for orbital invariants $\epsilon_{\alpha}$ and $l_{\alpha}$. Moreover, another representation, named the effective potential $\tilde{u} r$-diagram, complements the $\epsilon l$-diagram because it does not only reveal the constraints on orbital invariants but also shows the radial velocities along the orbits. These two latter diagrams themselves are also part of the kinetic features to be discussed.

\section{A. Orbital invariants $\epsilon$-diagram}

As shown in Fig. 1, for any $r$, three important boundaries $\left(l_{\alpha r}, l_{\alpha r}^{*}\right.$, and $\left.l_{r}^{*}\right)$ split the $\epsilon l$-plane into different domains. At the rightmost, one first finds the $l_{\alpha r}^{2}$ line. Evidently, this line moves with $r$ because $l_{\alpha r}$ depends on both $r$ and $\epsilon_{\alpha}$ [see Eq. (9)]. To construct the $l_{\alpha r}^{*}$ boundary at $r$, for any sufficient energy $\epsilon_{\alpha}>u_{\alpha r}^{*}$, the value of $l_{\alpha r}^{* 2}$ is found by taking the leftmost point of the $l_{\alpha r^{\prime}}^{2}$ lines by varying $r^{\prime}$ from the particle origin to $r$. Finally, the $l_{\alpha}^{*}$ boundary is found in a similar manner yet by varying $r^{\prime}$ from 1 to $\infty$, which is thus independent of $r$.

Since all three boundaries depend on the $r$-family of $l_{\alpha r}^{2}$ lines, we now examine some important features that reveal their variations with $r$. First of all, the slope

$$
\mathrm{d} \epsilon_{\alpha} / \mathrm{d} l_{\alpha r}^{2}=1 / r^{2}
$$

decreases with $r$. After using $F\left(r, \epsilon_{\alpha}, l_{\alpha}^{2}\right)=l_{\alpha}^{2}-r^{2}\left(\epsilon_{\alpha}-u_{\alpha r}\right)$ $=0$ to describe the $r$-family lines, the envelope that they slide on is found by $\partial F / \partial r=0$ as

$$
\epsilon_{\alpha, e n v}(r)=u_{\alpha r}+\frac{r \mathrm{~d} u_{\alpha r}}{2 \mathrm{~d} r}, \quad l_{\alpha, e n v}^{2}(r)=\frac{r^{3} \mathrm{~d} u_{\alpha r}}{2 \mathrm{~d} r} .
$$

Such an envelope is parametrized by $r$ and is tangent to the correspondent $l_{\alpha r}^{2}$ line at the point given by Eq. (27). Instead of plotting the full $r$-family lines, one just needs to plot the envelope in the $\epsilon l$-diagram. As will be demonstrated later in Secs. IV-VI, the critical $r$ locations-that separate the regions where the $l_{\alpha r}^{2}$ lines move in different manners - can be readily identified from such an envelope, for instance, see Fig. 4(b). Since the $r$ value correspondent to the corner of the envelope can be one of these critical $r$ locations, we look for the condition at the corner, which is given by $d \epsilon_{\alpha, e n v} / d r$ $=d l_{\alpha, e n v}^{2} / d r=0$ as [see Eqs. (6) and (27)]

$$
3 \frac{\mathrm{d} \varphi}{\mathrm{d} r}+\frac{r \mathrm{~d}^{2} \varphi}{\mathrm{d} r^{2}}=0,
$$

being dependent only on $\varphi(r)$ but not the species.

Interestingly, due to $d \epsilon_{\alpha, e n v} / d r=d l_{\alpha, e n v}^{2} / d r$ [see Eq. (27)], the envelope can only move north-east or south-west as $r$ varies. Also, if the envelope lies outside the first quadrant, the $l_{\alpha r}^{2}$ lines cannot cross each other inside this quadrant. Therefore, the lines always move to the left (right) in the first quadrant for decreasing (increasing) $r$ as plasma particles (emitted electrons) head to the probe (infinity). If the envelope enters the first quadrant, the variation of the $l_{\alpha r}^{2}$ lines becomes more complicated [see Secs. IV-VI].

\section{B. Effective potential ũ r-diagram}

The normalized effective potential is defined as ${ }^{13,18}$

$$
\tilde{u}_{\alpha r}\left(r, l_{\alpha}^{2}\right) \equiv u_{\alpha r}+l_{\alpha}^{2} / r^{2}=\epsilon_{\alpha}-v_{r}^{2} .
$$

For one $l_{\alpha}^{2}$ value, a $\tilde{u}_{\alpha r}$-versus- $r$ curve can be drawn and they move upwards with $l_{\alpha}^{2}$. Such a diagram is helpful because, according to Eq. (29), the vertical distance from the $\tilde{u}_{\alpha r}$ curve to the horizontal line $\tilde{u}_{\alpha r}=\epsilon_{\alpha}$ directly gives $v_{r}^{2}$. A possible $\tilde{u} r$-diagram is shown qualitatively in Fig. 2, which will be used for the discussions of all species (although each species has its own $\tilde{u} r$-diagram).

As observed in Fig. 2, a plasma particle of energy $\epsilon_{1}$ and angular momentum $l_{1}$ moves inwards from infinity with decreasing radial velocity. This velocity vanishes at $r_{1}$ as $\tilde{u}_{\alpha r}\left(r_{1}, l_{1}^{2}\right)=\epsilon_{1}$. The particle is then reflected back to infinity. Therefore, for the energy $\epsilon_{\alpha}$, the effective potential barrier at $r_{1}$ (owing to orbital effects) sets a limit on the angular momentum as $l_{i} \leq l_{1}=l_{\alpha r}\left(\epsilon_{1}, r_{1}\right)=l_{\alpha r}^{*}\left(\epsilon_{1}, r_{1}\right)$. An emitted electron with the same angular momentum and energy actually cannot be emitted from the probe, due to $\tilde{u}_{\alpha r}\left(1, l_{1}^{2}\right)>\epsilon_{1}$ and $l_{1}>l_{\alpha r}\left(\epsilon_{1}, 1\right)$. However, if such an electron has a lower angular momentum, say $l_{2}$, it is emitted at the probe and reflected back at $r_{2}$, due to $\tilde{u}_{\alpha r}\left(1, l_{2}^{2}\right)<\epsilon_{1}, \tilde{u}_{\alpha r}\left(r_{2}, l_{2}^{2}\right)=\epsilon_{1}$, and $l_{2}=l_{\alpha r}\left(\epsilon_{1}, r_{2}\right)=l_{\alpha r}^{*}\left(\epsilon_{1}, r_{2}\right)<l_{\alpha r}\left(\epsilon_{1}, 1\right)$. Consequently, for some further location, say $r_{m}$, even though such an electron is allowed by the non-forbidden condition as $\epsilon_{1}>\tilde{u}_{\alpha r}\left(r_{m}, l_{2}^{2}\right)$, it still cannot reach $r_{m}$ as prohibited by the sufficient condition, i.e., $l_{\alpha, r}\left(\epsilon_{1}, r_{m}\right)>l_{2}>l_{\alpha, r}^{*}\left(\epsilon_{1}, r_{m}\right)=l_{3}$. If the $\tilde{u}_{\alpha r}$ curve for a given $l_{\alpha}$ exhibits a minimum, see Fig. 4(a) for instance, the particle with angular momentum $l_{\alpha}$ and energy $\epsilon_{\alpha}$ is trapped between the two radii where the $\tilde{u}_{\alpha r}$ curve intersects the horizontal $\epsilon_{\alpha}$ line.

In Sec. III A, the $r$-family lines in the $\epsilon l$-diagram can be substituted by a single curve-the envelope. Similarly in the $\tilde{u} r$-diagram, the $l$-family curves can be substituted by just two geometric elements. The first one is the effective potential curve for zero angular momentum

$$
\tilde{u}_{\alpha r}(r, 0)=u_{\alpha r}(r)
$$

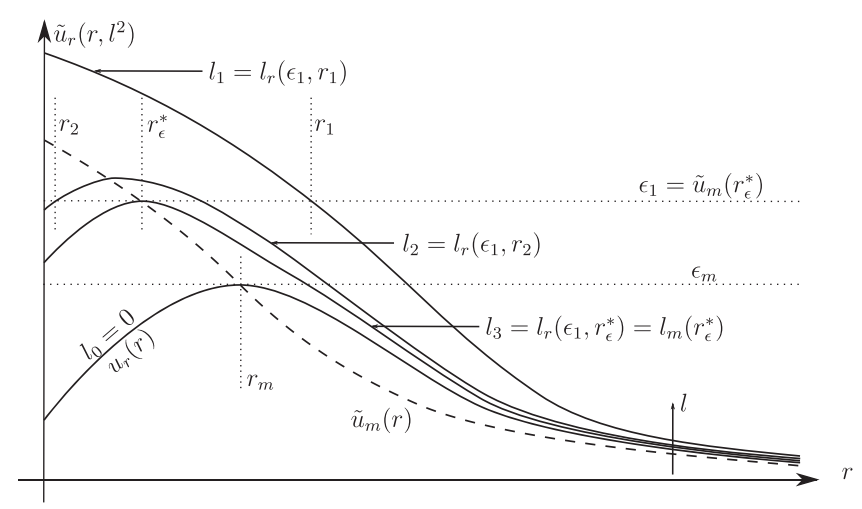

FIG. 2. An $l$-family of $\tilde{u}_{\alpha r}$ (solid) curves and the extreme (dashed) curve $\tilde{u}_{\alpha m}(r)$ is shown qualitatively as a possible $\tilde{u} r$-diagram. The subscript $\alpha$ is omitted in the plot. 
which coincides with the electric potential energy. The second element arises from the fact that the $\tilde{u}_{\alpha r}$ curves can be non-monotonic in $r$, exhibiting maxima and minima. For this reason, instead of the full $l$-family curves, we will pay attention to the curve made of their local extreme points. Such an extreme curve is found from $\partial \tilde{u}_{\alpha r} / \partial r=0$, giving $l_{\alpha m}^{2}(r)$ $=r^{3} \partial \tilde{u}_{\alpha r} / 2 \partial r$. This value coincides with the one found for the envelope [see $l_{\alpha, e n v}^{2}$ in Eq. (27)] and the extreme curve (the dashed curve in Fig. 2) is thus given by

$$
\begin{gathered}
\tilde{u}_{\alpha m}(r)=\tilde{u}_{\alpha r}\left(r, l_{\alpha m}^{2}\right)=\epsilon_{\alpha, e n v}(r), \\
l_{\alpha, m}^{2}(r)=l_{\alpha, e n v}^{2}(r) .
\end{gathered}
$$

The extreme curve and the envelope are therefore connected and can relate the $\epsilon l$-diagram and the $\tilde{u} r$-diagram.

The $\tilde{u} r$-diagram can also be used to illustrate the absorption radius concept in the probe theory. For a given horizontal $\epsilon_{\alpha}$ line, it possibly intersects with the extreme curve at a location $r_{\epsilon}^{*}$, for instance, $\tilde{u}_{\alpha m}\left(r_{\epsilon}^{*}\right)=\epsilon_{1}$ in Fig. 2. Such $r_{\epsilon}^{*}$ can be the absorption radius for that energy if the $\tilde{u}_{\alpha r}$ curve for the associated angular momentum given by Eq. (31) crosses the extreme curve with a maximum, for instance, $l_{\alpha m}\left(r_{\epsilon}^{*}\right)$ $=l_{3}=l_{\alpha r}\left(\epsilon_{1}, r_{\epsilon}^{*}\right)$ in Fig. 2. If we take an emitted electron and Fig. 2 as an examples, physically it means that, for the energy $\epsilon_{1}$ and at any further location $r^{\prime}>r_{\epsilon}^{*}$, the angular momentum limit for an electron to arrive at $r^{\prime}$ is set by the orbital barrier at the absorption radius $r_{\epsilon}^{*}$ as $l_{\alpha r}^{*}\left(\epsilon_{1}, r^{\prime}\right)=l_{\alpha m}\left(r_{\epsilon}^{*}\right)$, instead of at $r^{\prime}$ due to $l_{\alpha m}\left(r_{\epsilon}^{*}\right)<l_{\alpha r}\left(\epsilon_{1}, r^{\prime}\right)$.

\section{Distribution function $\epsilon_{r} \epsilon_{\theta}$ diagram}

For a given $r$ location, the local distribution function of particles is normally presented in a $v_{r} v_{\theta}$-plane. However, in this work, we will follow previous studies ${ }^{22-24}$ and use the directional-energy $\epsilon_{r} \epsilon_{\theta}$-plane (see Fig. 3):

$$
\epsilon_{r}=\frac{\epsilon_{\alpha} v_{r}}{\sqrt{v_{r}^{2}+v_{\theta}^{2}}}, \quad \epsilon_{\theta}=\frac{\epsilon_{\alpha} v_{\theta}}{\sqrt{v_{r}^{2}+v_{\theta}^{2}}} .
$$

This is convenient because, on such a plane, the radial distance from a point to the origin is the total energy of a

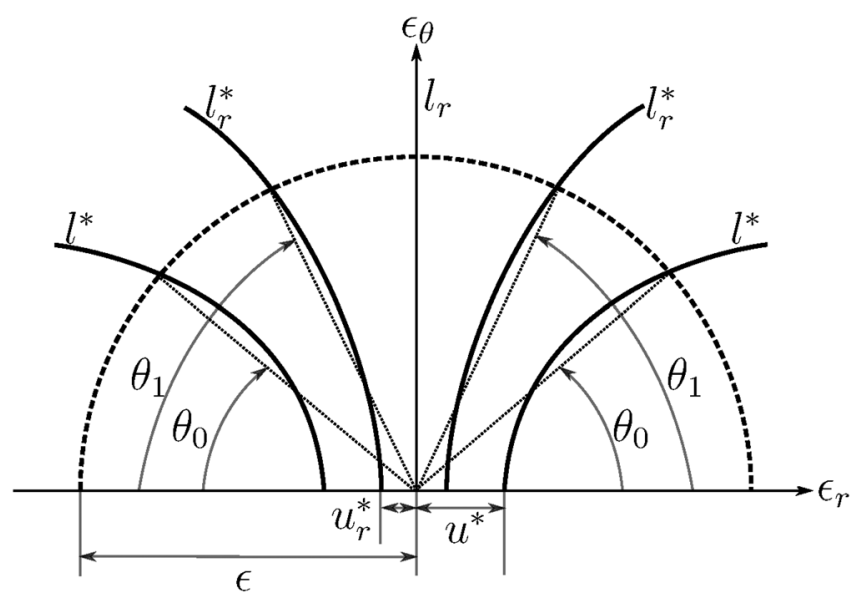

FIG. 3. Boundaries in the directional-energy plane for $\epsilon_{\theta}>0$ (symmetric to $\left.\epsilon_{\theta}<0\right)$. particle, $\epsilon_{\alpha}^{2}=\epsilon_{r}^{2}+\epsilon_{\theta}^{2}$. The azimuthal angle $\theta$ from the horizontal axis is related to the angular momentum as

$$
\sin \theta=\left|\epsilon_{\theta}\right| / \epsilon_{\alpha}=l_{\alpha} / l_{\alpha r}
$$

which is defined only for $0<\theta<\pi / 2$ as shown in Fig. 3 .

Eventually, for a given $r$, the energy and angular momentum constraints found in the $\epsilon l$-plane are equivalent to the limitations on radial $\left(\epsilon_{\alpha}\right)$ and azimuthal $(\theta)$ coordinates of the $\epsilon_{r} \epsilon_{\theta}$-plane. The three boundaries- $l_{\alpha r}, l_{\alpha r}^{*}$, and $l_{\alpha}^{*}$-in Fig. 1 thus also appear in Fig. 3. First of all, the $l_{\alpha r}$ boundary collapses with the $\epsilon_{\theta}$-axis due to $l_{\alpha} \rightarrow l_{\alpha r}$ as $\theta \rightarrow \pi / 2$. Therefore, the forbidden domain, a region that we are not interested in, is directly excluded from the $\epsilon_{r} \epsilon_{\theta}$-plane, because the populated regions in local distribution functions are constraint by sufficient and captured conditions. The $l_{\alpha r}^{*}$ and $l_{\alpha}^{*}$ boundaries appear in Fig. 3 (for $\epsilon_{\alpha} \geq u_{\alpha r}^{*}$ and $\epsilon_{\alpha} \geq u_{\alpha}^{*}$ ) as limitations on $\theta$ for an $\epsilon_{\alpha}$ radius, being

$$
\sin \theta_{1}=l_{\alpha r}^{*} / l_{\alpha r} \quad \text { and } \quad \sin \theta_{0}=l_{\alpha}^{*} / l_{\alpha r},
$$

respectively. If there is no electric potential barrier anywhere $\left(u_{\alpha r}^{*}=u_{\alpha}^{*}=0\right)$, the holes (unpopulated regions) in the distribution functions can only be bound by the sufficient boundary $l_{\alpha r}^{*}$ and/or the captured boundary $l_{\alpha}^{*}$ [see Fig. 4(c) as an example]. Otherwise, the energy limitation $u_{\alpha r}^{*}$ can possibly appear as a circle that bounds a centred hole region [see Fig. $8(c)$ as an example]. To conclude, in distribution functions, there can only be three kinds of holes that are bound by the sufficient boundary $l_{\alpha r}^{*}$, the captured boundary $l_{\alpha}^{*}$, and the electric potential barrier $u_{\alpha r}^{*}$.

In the $\epsilon_{r} \epsilon_{\theta}$-plane, for the regions where particles do exist at $r, \mathrm{~d} \epsilon_{r} \mathrm{~d} \epsilon_{\theta}=\mathrm{d} v_{r} \mathrm{~d} v_{\theta}$ gives the local directional-energy distribution function as

$$
f_{\alpha}\left(r, \epsilon_{r}, \epsilon_{\theta}\right)=f_{\alpha}\left(r, v_{r}, v_{\theta}\right)=H_{\alpha} \exp \left(-\epsilon_{\alpha}\right) / \pi .
$$

For the regions that are unpopulated by particles, holes appear as $f_{\alpha}=0$. Because $\epsilon_{\alpha}$ is the radial coordinate of the $\epsilon_{r} \epsilon_{\theta}$-plane and $f_{\alpha}$ is independent of $\theta$, the distribution function takes the same value (if not zero) on a circle centred at the origin of the plane. Moreover, for distribution functions at different $r$, since $f_{\alpha}$ is invariant with $r$, the circles with the same $\epsilon_{\alpha}$ radius also share the same $f_{\alpha}$ value. Due to these advantages of the $\epsilon_{r} \epsilon_{\theta}$-diagram, the density variation along $r$ can then be straightforwardly discussed by looking at the area of the populated region in directional-energy distribution functions.

According to Eq. (32), the $\epsilon_{r}>0$ plane corresponds to outgoing particles $\left(\epsilon_{r}<0\right.$ for incoming). Therefore, the populated region in the $\epsilon_{r}>0$ plane represents the second-arriving $D_{2 r}$ domain for plasma particles and the first-arriving $D_{1 r}$ domain for emitted electrons (vice versa for $\epsilon_{r}<0$ ). The difference in both planes amounts to the captured domain, $D_{0}=D_{1 r}-D_{2 r}$, which is bound by the $l_{\alpha}^{*}$ boundary.

In Secs. IV-VI, the distribution at some representative $r$ locations will be shown for $\epsilon_{\alpha} \leq 2$ by using pseudocolor maps, with the color scaled between zero and $H_{\alpha} / \pi$. Macroscopic quantities will also be shown together with $\epsilon l$-diagrams and $\tilde{u} r$-diagrams. Guided by the envelope in the $\epsilon l$-diagram, how the three boundaries $\left(l_{\alpha r}, l_{\alpha r}^{*}, l_{\alpha}^{*}\right)$ vary with $r$ 
can be visualized straightforwardly. In local distribution $\epsilon_{r} \epsilon_{\theta}$-diagrams, because these boundaries can be related to radial $\left(u_{\alpha r}^{*}\right.$ and $\left.u_{\alpha}^{*}\right)$ and angular $\left(\theta_{0}\right.$ and $\left.\theta_{1}\right)$ limits, how the unpopulated holes vary with $r$ can be discussed, which then reveals the density variation. At the end, with the radial velocity $v_{r}$ shown in the $\tilde{u} r$-diagram, kinetic temperatures can also be discussed.

\section{MONOTONIC POTENTIAL, $\varphi_{p}<0$}

Our first case of study corresponds to the parameter values $\rho_{0}=1, \delta_{i}=1, \delta_{\text {em }}=0.34, \beta=11.81$, and $\varphi_{p}=-40$. The numerical solution of Eq. (3) gives a monotonic potential profile, with ion collection being OML (operational regime 6 in Ref. 21). By solving Eq. (27) with $\varphi(r)$ found numerically, the envelope in the $\epsilon$-diagram is found for each species. As given by Eq. (28), the envelope has two corners at $r=7.47$ and 16.96 , being independent of the species.

\section{A. Plasma ions}

As shown in Fig. 4(c), there are only two types of holes in the local distributions. One type, which crosses and is symmetric to the $\epsilon_{r}$-axis, represents the ions that are captured by the probe. These holes are bound by the $l_{\alpha}^{*}$ boundary and occur at all $r$ locations for all energies $\epsilon_{i} \geq 0$. Because the captured ions exist at $r$ as incoming particles but not as outgoing particles, these holes appear only in the $\epsilon_{r}>0$ plane. For any energy $\epsilon_{i}$, the $\theta$ limit of such a hole is given by $\theta_{0}$ in Eq. (34). The other type, which crosses and is symmetric to the $\epsilon_{\theta}$-axis, represents the ions that are reflected back to infinity due to the existence of absorption radius for some energies and thus bound by the $l_{\alpha r}^{*}$ boundary. These holes occur only for some $r$ and for energies below certain threshold. For any of these energies, the $\theta$ limit is given by $\theta_{1}$ in Eq. (34). For $1.16 \leq r \leq 13.63$, the second type of hole appears and the energy threshold is maximum at $r=7.47$. These three $r$ locations divide the space into four distinct regions where the ion kinetic features behave differently. These features will be discussed separately in the four regions, from infinity to probe. The envelope in Fig. 4(b) serves as a simple guide to understand the variation of the holes.

\section{Without absorption radius region ( $r>13.63)$}

For ions that move from infinity to probe (decreasing $r$ ), we need to read the envelope in Fig. 4(b) by following the arrows that start from the bottom right. For a cylindrical probe, one has $u_{i r} \sim-1 / r$ at infinity. ${ }^{13}$ Given by Eq. (27), the envelope lies below the first quadrant at infinity and moves south-west in Fig. 4(b) as $r$ decreases. At $r=16.96$, it reaches a corner and then continues to move north-east until crossing $\epsilon_{i}=0$ at $r=13.63$. The $l_{i r}^{2}$ lines slide on the envelope and always move to the left in the first quadrant. Therefore, for any energy $\epsilon_{i} \geq 0$, the value of $l_{i r}$ decreases as $r$ decreases. Consequently, the non-forbidden condition is also sufficient for ions to arrive at $r$ and there is no any absorption radius for any energy, i.e., $l_{i r}^{*}=l_{i r}$ for $\epsilon_{i} \geq 0$. Such a condition can also be observed from the $\tilde{u} r$-diagram in Fig. 4(a), in which all $\tilde{u}_{i r}$ curves for effective potential are monotonic in the $\tilde{u}_{i r}>0$ plane for $r>13.63$.

Shown by any local distribution function in Fig. 4(c) for $r>13.63$, there is only one hole due to captured ions. For any energy $\epsilon_{i}>0$ and a decreasing $r$, the $\theta_{0}=\arcsin \left(l_{i}^{*} / l_{i r}\right)$ limit increases, because $l_{i}^{*}$ is independent of $r$ [see Eq. (13)] while $l_{i r}$ reduces [see Fig. 4(b)]. Therefore, this hole enlarges and the density drops in Fig. 4(c). The other type of hole bound by $l_{i r}^{*}$ does not appear, due to $l_{i r}^{*}=l_{i r}$ and $\theta_{1}=\arcsin \left(l_{i r}^{*} / l_{i r}\right)=\pi / 2$. From the inset of the $\tilde{u} r$-diagram in Fig. 4(a), the radial velocity $v_{r}$ is found to increase for lower $l_{i}$ values yet decrease for higher ones. Such a dispersion in $v_{r}$ explains the rise of the kinetic temperature $\tilde{T}_{i r}$ in Fig. 4(c). The $\tilde{T}_{i \theta}=\left\langle v_{\theta}^{2}\right\rangle$ curve also rises because ions are accelerated in the azimuthal direction (except $l_{i}=0$ ) following angular momentum conservation. This increasing behaviour for $\tilde{T}_{i \theta}$ remains for the two following $r$-regions.
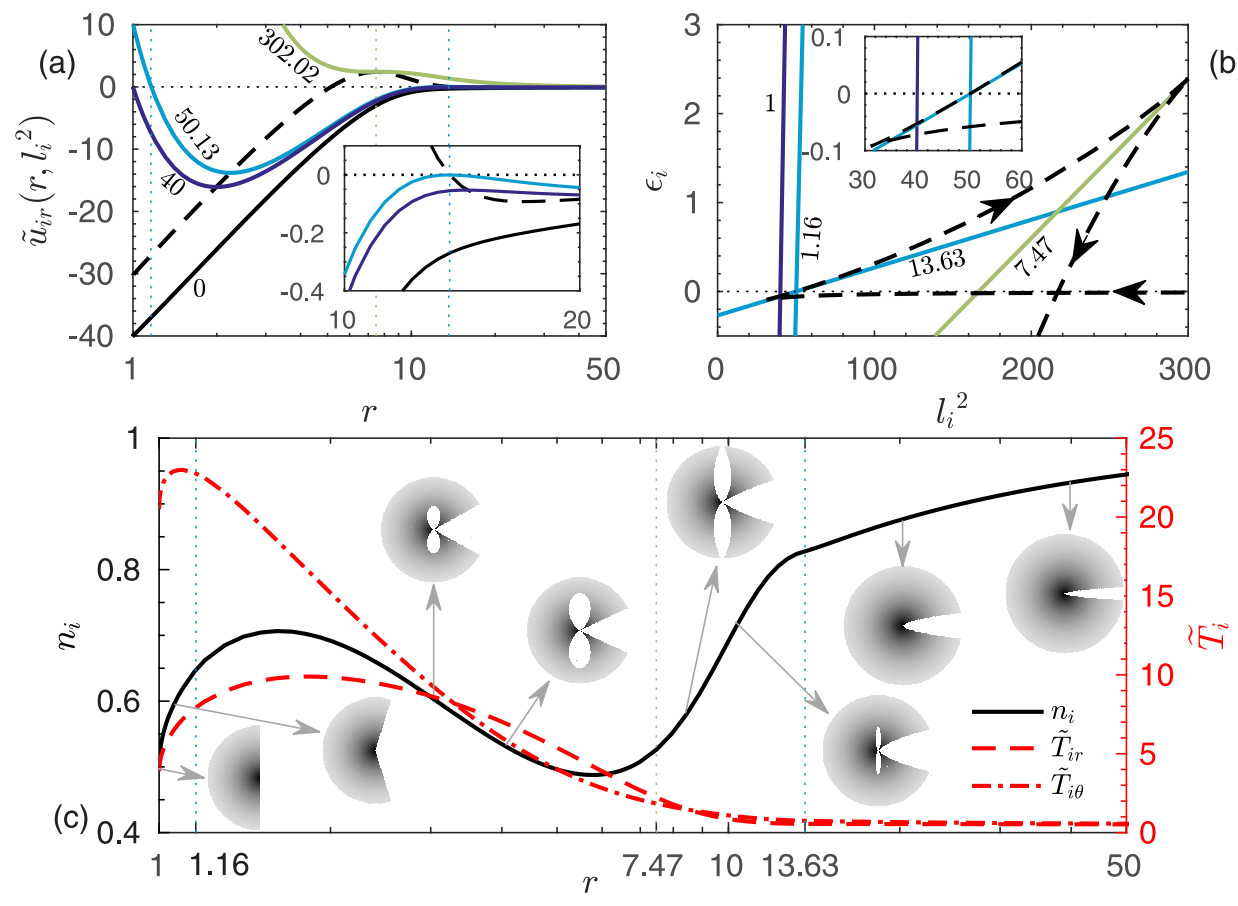

FIG. 4. For $\rho_{0}=1, \delta_{i}=1, \delta_{e m}=0.34$, $\beta=11.81, \varphi_{p}=-40$, and plasma ions: (a) the $\tilde{u} r$-diagram, with the dashed curve for $\tilde{u}_{i m}$ and the solid curves for $\tilde{u}_{i r}$ at different $l_{i}^{2}$ values; (b) the $\epsilon l$-diagram, with the dashed curve for the envelope and the solid lines for $l_{i}^{2}=l_{i r}^{2}$ at different $r$ values; (c) density profile, kinetic temperature profiles $\tilde{T}_{r}$ and $\tilde{T}_{\theta}$, and the directional distribution for $\epsilon_{i} \leq 2$. 


\section{With absorption radius region $I(7.47<r<13.63)$}

At $r=13.63$, the envelope enters the first quadrant and moves north-east until the second corner at $r=7.47$. For a given $r$, it becomes a bit more complicated to find the $l_{i r}^{*}$ boundary following its definition in Eq. (12), for which the energy range needs to separated by $\epsilon_{i, \text { env }}(r)$. At $\epsilon_{i}=\epsilon_{i, \text { env }}(r)$, the $l_{i r}^{2}$ line is tangent to the envelope. For $\epsilon_{i}>\epsilon_{i, e n v}(r)$, this line is to the left of any $l_{i r^{\prime}}^{2}$ line for $r^{\prime}>r$ and the non-forbidden condition is thus sufficient as $l_{i r}^{*}=l_{i r}$. Nevertheless, for $\epsilon_{i}<\epsilon_{i, e n v}(r)$, the $l_{i r}^{2}$ line crosses some $l_{i r^{\prime}}^{2}$ lines in the first quadrant and lies at the right below the intersection. For any energy $\epsilon_{i}<\epsilon_{i, e n v}(r)$, the leftest point of the $l_{i r^{\prime}}^{2}$ lines is found in Fig. 4 (b) by the intersection of the envelope with the horizontal $\epsilon_{i}$ line. The value of $l_{i r}^{*}$ for that energy can thus be evaluated by the envelope at the absorption radius $r_{\epsilon}^{*}$ as $l_{i r}^{*}\left(r, \epsilon_{i}\right)$ $=l_{i, e n v}\left(r_{\epsilon}^{*}\right)$, with $r_{\epsilon}^{*}$ given by the condition $\epsilon_{e n v}\left(r_{\epsilon}^{*}\right)=\epsilon_{i}$.

For the distribution functions in Fig. 4(c), the holes caused by captured ions (limited by $\theta_{0}$ ) are still present, but with a more complex behaviour compared with the previous $r$-region. In addition to them, the other kind of holes-due to absorption radius and limited by $\theta_{1}$-appears for $\epsilon_{i}$ $<\epsilon_{i, e n v}(r)$, crossing the $\epsilon_{\theta}$-axis. Now we analyse the variation of the holes for two different energy ranges separated by $\epsilon_{i, e n v}(r)$-a value that increases with decreasing $r$. For $\epsilon_{i}<\epsilon_{i, \text { env }}(r)$, at a given radius $r$, the $l_{i r}^{2}$ line tends to move to the right towards the line for a smaller $r$. Therefore, both $\theta_{0}=\arcsin \left(l_{i}^{*} / l_{i r}\right)$ and $\theta_{1}=\arcsin \left(l_{i r}^{*} / l_{i r}\right)$ would decrease due to constant $l_{i}^{*}$ and $l_{i r}^{*}=l_{i, e n v}\left(r_{\epsilon}^{*}\right)$. As a consequence, in the $\epsilon_{r}<0$ plane, the populated region (limited only by $\theta_{1}$ ) grows smaller. In the $\epsilon_{r}>0$ plane, the populated region (limited by both $\theta_{0}$ and $\theta_{1}$ ) also grows smaller due to

$$
\begin{aligned}
& -\mathrm{d}\left(\theta_{1}-\theta_{0}\right) / \mathrm{d} r \\
& =\frac{\mathrm{d} l_{i r} / \mathrm{d} r}{l_{i r}}\left(\frac{1}{\sqrt{l_{i r}^{2} / l_{i r}^{* 2}-1}}-\frac{1}{\sqrt{l_{i r}^{2} / l_{i}^{* 2}-1}}\right)<0,
\end{aligned}
$$

which has the same sign as $\mathrm{d} l_{i r} / \mathrm{d} r$, being smaller than zero. For $\epsilon_{i r}>\epsilon_{i, e n v}(r)$, the holes bound by the $l_{i r}^{*}$ boundary disappears due to $\theta_{1}=\pi / 2$ and $l_{i r}^{*}=l_{i r}$. Since the $l_{i r}^{2}$ line tends to move to the left for these energies, the populated region also grows smaller with increasing $\theta_{0}=\arcsin \left(l_{i}^{*} / l_{i r}\right)$. Compared to the previous $r$-region, more reduction in the overall populated region thus results in a steeper drop of density in Fig. 4 (c). For the kinetic temperature $\tilde{T}_{i r}$, because the $\tilde{u}_{i r}$ curves in Fig. 4(a) are more separated, more dispersion in $v_{r}$ leads to a faster rise of $\tilde{T}_{i r}$ in Fig. 4(c).

\section{With absorption radius region II $(1.16<r<7.47)$}

From $r=7.47$, the last branch of the envelope in Fig. 4 (b) moves from the second corner south-west. At any location $r$, the $l_{i r}^{2}$ line osculates this branch at $\epsilon_{i, e n v}(r)$ and crosses the second branch (between the two corners) at another energy $\epsilon_{c}>\epsilon_{i, e n v}(r)$. At $r=1.16$, the $l_{i r}^{2}$ line crosses the second branch with $\epsilon_{c}=0$. To find the $l_{i r}^{*}$ boundary at $r$, the energy is again separated into two regions, but this time by $\epsilon_{c}$. For $\epsilon_{i}>\epsilon_{c}$, the non-forbidden condition is sufficient as $l_{i r}^{*}=l_{i r}$. For $\epsilon_{i}<\epsilon_{c}$, with the second branch of the envelope lying to the left of the $l_{i r}^{2}$ line, it still sets the $l_{i r}^{*}$ boundary as $l_{i r}^{*}\left(r, \epsilon_{i}\right)=l_{i, e n v}\left(r_{\epsilon}^{*}\right)\left(7.47<r_{\epsilon}^{*}<16.63\right)$.

As shown in Fig. 4(c), the ion density exhibits a minimum and a maximum for $1.16<r<7.47$. This behaviour is directly related to the variation of both kinds of holes in distribution functions. The energy threshold for the hole limited by $\theta_{1}$ is given by $\epsilon_{c}$, instead of $\epsilon_{i, e n v}(r)$ for the previous $r$-region. The value of $\epsilon_{c}$ decreases from $\epsilon_{c}=\epsilon_{i, e n v}(r)$ at $r=7.47$ to zero at $r=1.16$. For $\epsilon_{i}>\epsilon_{c}$, there is only the hole due to captured ions. The $\theta_{0}=\arcsin \left(l_{i}^{*} / l_{i r}\right)$ limit increases because the $l_{i r}^{2}$ line moves to the left for decreasing $r$. The populated region for these energies thus grows smaller. For $\epsilon_{i}<\epsilon_{c}$, the $\theta_{1}$ limit appears and the variation of the populated region has to be discussed for two different cases: (a) if $\epsilon_{i, e n v}(r)>0$, the $l_{i r}^{2}$ line for $\epsilon_{i, e n v}(r)<\epsilon_{i}<\epsilon_{c}$ would move to the left, which enlarges the populated region due to increasing $\theta_{1}$ and $\theta_{1}-\theta_{0}$ [see Eqs. (34) and (36)], yet the contrary happens for $\epsilon_{i}<\epsilon_{i, e n v}(r)$; (b) if $\epsilon_{i, \text { env }}(r)<0$, the populated region grows bigger for all $\epsilon_{i}<\epsilon_{c}$. At the end, whether the density would increase or decrease depends on the change of the populated region for the dominant low energy range (thus high $f_{\alpha}$ ). As ions move inwards, the density thus decreases to a minimum, increases to a maximum, and decreases again because the dominant range is $0<\epsilon_{i}$ $<\epsilon_{i, e n v}(r), 0<\epsilon_{i}<\epsilon_{c}$, and $\epsilon_{i}>\epsilon_{c}$, respectively. The kinetic temperature $\tilde{T}_{i r}$ in Fig. 4(c) is also not monotonic and exhibits a maximum. This can be explained by looking at the $\tilde{u}_{i r}$ curves in Fig. 4(a). For $1.16<r<7.47$, some of them cross the dashed extreme curve $\tilde{u}_{i m}$ with a minimum. Due to the presence of these minima, the dispersion in $v_{r}$ is reduced close to $r=1.16$ and $\tilde{T}_{i r}$ thus drops.

\section{Without absorption radius region $(1<r<1.16)$}

In the $\epsilon l$-diagram in Fig. 4 (b), the $l_{i r}^{2}$ lines slide on the envelope that is outside the first quadrant. In addition, since they lie to the left of any envelope in the first quadrant, the non-forbidden condition is again sufficient as $l_{i r}^{*}=l_{i r}$ for all $\epsilon_{i} \geq 0$. In distribution functions, there is thus only one hole due to captured ions. The populated region grows smaller towards the probe due to increasing $\theta_{0}$. Therefore, the ion density drops, until being half at the probe as given by the OML condition. For kinetic temperatures, $\tilde{T}_{i r}$ continues dropping. In the azimuthal direction, $\tilde{T}_{i \theta}$ first increases, yet decreasing near the probe. This decrease is because of the loss of ions with high $\left|v_{\theta}\right|\left(l_{i}>l_{i r}\right.$ due to orbital effects), which causes a reduction in $\left\langle v_{\theta}^{2}\right\rangle$ that becomes more than the increase introduced by angular momentum conservation.

To conclude, ion collection is reduced by orbital effects that forbid ions with large angular momenta. For some energies, the orbital barrier at the absorption radius $\left(7.47<r_{\epsilon}^{*}\right.$ $<16.63)$ reduces the amount of ions at smaller $r$, by excluding the ions with $l_{\alpha, e n v}\left(r_{\epsilon}^{*}\right)<l_{\alpha r}$. The energy threshold $\left(\epsilon_{i, \text { env }}\right.$ or $\epsilon_{c}$ )-below which there is absorption radius-first increases and then reduces, yielding the non-monotonic density in Fig. 4(c). Because this energy threshold reduces to zero before ions reaching the probe, the ion collection in this case is thus OML for all $\epsilon_{i} \geq 0$, with the amount of captured ions being limited by the local orbital barrier at the probe 
only $\left(l_{i}^{*}=l_{i R}\right)$. The distribution at the probe is then HM and covers the entire $\epsilon_{r}<0$ plane.

If one reduces the probe bias $\varphi_{p}$, ion collection can be non-OML (regime 7 in Ref. 21). For non-OML collection, at the probe, there are still absorption radii for some energies $\left(l_{i}^{*}<l_{i R}\right)$ and holes would appear in the $\epsilon_{r}<0$ plane-bound by the $l_{i R}^{*}$ boundary and crossing the $\epsilon_{\theta}$ axis. This reduces the current collected by the probe, being smaller than the OML current - the maximum current can be collected by a cylindrical probe for a given probe bias. However, a further decrease in $\varphi_{p}$ can result in another transition to OMLcollection (regime 8 in Ref. 21).

For plasma ions, due to $u_{i r}<0$, there is no electric potential barrier anywhere and only the angular momentum constraints need to be discussed. However, for plasma electrons, as shown in Sec. IV B, the energy constraints also need to be taken into account.

\section{B. Plasma electrons}

As predicted, the envelope in the $\epsilon l$-diagram in Fig. 5(b) also has two corners at $r=7.47$ and 16.96 for plasma electrons. Guided by the arrows from infinity to probe, the envelope is above the $\epsilon_{e}=0$ axis for $r \rightarrow \infty\left(u_{e r} \sim 1 / r\right)$ and moves north-east until the first corner, after which it extends south-west until the second corner and then north-east again. However, with the envelope being entirely outside the first quadrant, the discussion of kinetic features becomes much easier for plasma electrons. Because the slope of $l_{e r}^{2}$ lines increases with reducing $r$, these lines can only move to the left in the first quadrant. For a given $r$, the $l_{e r}^{2}$ line intersects with the positive $\epsilon_{e}$-axis at the potential energy $u_{e r}>0$. Since $u_{e r}$ increases towards the probe, energy constraints appear due to electric potential barrier $u_{e r}^{*}=u_{e r}$. For energies below this constraint $\left(\epsilon_{e}<u_{e r}\right)$, the distribution function vanishes. For higher energies $\epsilon_{e}>u_{e r}$, the non-forbidden condition is sufficient as $l_{e r}^{*}=l_{e r}$.
In this case, two kinds of holes exist in distribution functions. The kind shown in Fig. 5(c) results from the vanished $f_{\alpha}$ for $\epsilon_{e}<u_{e r}$ due to electric potential barriers. They appear as circles centred at the origin of the $\epsilon_{r} \epsilon_{\theta}$-plane. Such a hole grows bigger with increasing $u_{e r}$ as electrons move inwards. Another kind of holes due to captured electrons, although not shown in Fig. 5(c), exists in the $\epsilon_{r}>0$ plane but only for very high energies $\epsilon_{e}>u_{e R}$. These holes cross the $\epsilon_{r}$-axis and are bound by the $l_{e R}$ boundary, which also grows bigger as $\theta_{0}$ increases with decreasing $l_{e r}$. At the end, because both kinds of holes grow bigger all the way towards the probe, the density drops.

It is interesting to note that for $u_{e r}<\epsilon_{e}<u_{e R}$, full circles are covered without any hole. The $\epsilon_{r}<0$ plane is entirely covered due to the fact that all electrons allowed by the non-forbidden condition can arrive at $r$ as incoming electrons, and thus, $\theta_{1}=\pi / 2$. Because all these electrons cannot overcome the electric potential barrier at the probe $\left(\epsilon_{e}\right.$ $\left.<u_{e R}\right)$, they are reflected back and cover the entire $\epsilon_{r}>0$ plane as outgoing particles.

In the $\tilde{u} r$-diagram in Fig. 5(a), the extreme curve $\tilde{u}_{e m}$ lies below all $\tilde{u}_{e r}$ curves $\left(\tilde{u}_{e m}<\tilde{u}_{e r}\right)$ due to $\mathrm{d} u_{e r} / \mathrm{d} r<0$ [see Eqs. (31a) and (27)]. All $\tilde{u}_{e r}$ curves thus increase monotonically towards the probe (instead of crossing $\tilde{u}_{e m}$ with local extrema). Therefore, the radial velocities of all electrons decrease towards the probe and $\tilde{T}_{e r}$ drops in Fig. 5(c). However, $\tilde{T}_{e \theta}$ first rises from being $1 / 2$ at infinity and then drops to be $1 / 2$ again at the probe. Similar to that explained for ions, the increase owes to angular momentum conservation and the decrease results from orbital effects. The variation of $\tilde{T}_{e \theta}$ is found to be much less than that of $\tilde{T}_{i \theta}$ in Fig. 4 (a), due to the fact that the electric potential barriers for electrons reduce the kinetic energy and thus suppress the increase of $\left|v_{\theta}^{2}\right|$.

\section{Emitted electrons}

The $\tilde{u} r$-diagram and the $\epsilon$-diagram are not presented here for emitted electrons because they can be simply
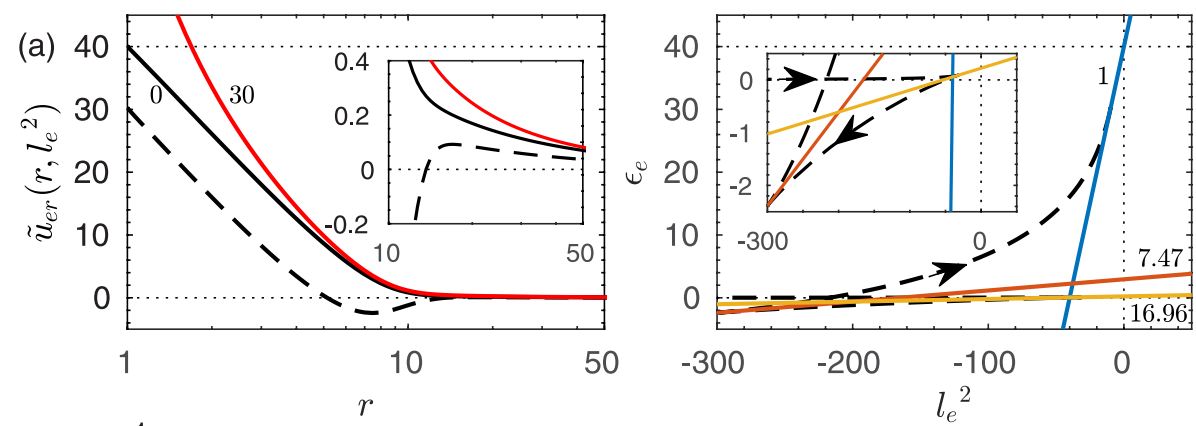

(b) (c)

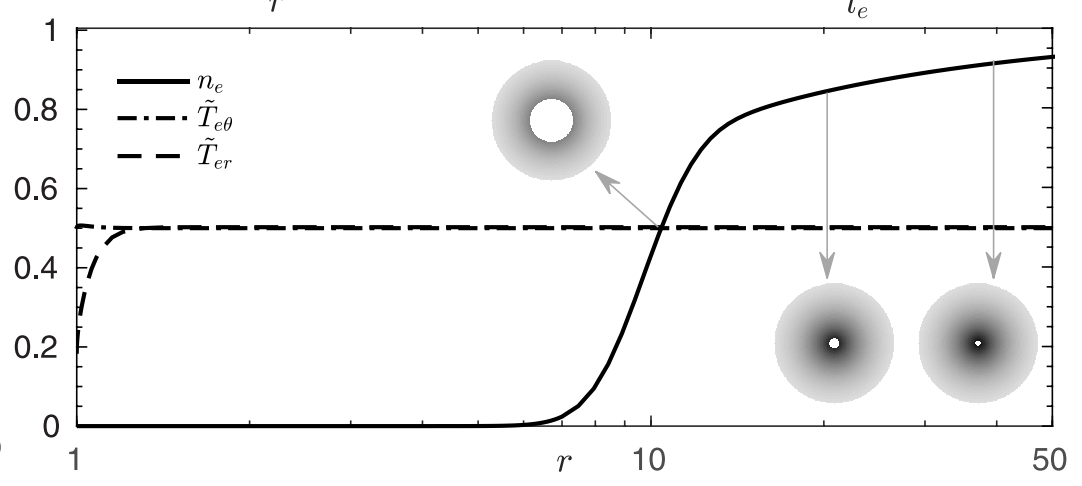

FIG. 5. For $\rho_{0}=1, \delta_{i}=1, \delta_{\text {em }}=0.34$, $\beta=11.81, \varphi_{p}=-40$, and plasma electrons: (a) $\tilde{u} r$-diagram; (b) $\epsilon l$-diagram; (c) density profile, kinetic temperature profile, and the directional distribution for $\epsilon_{e} \leq 2$. 


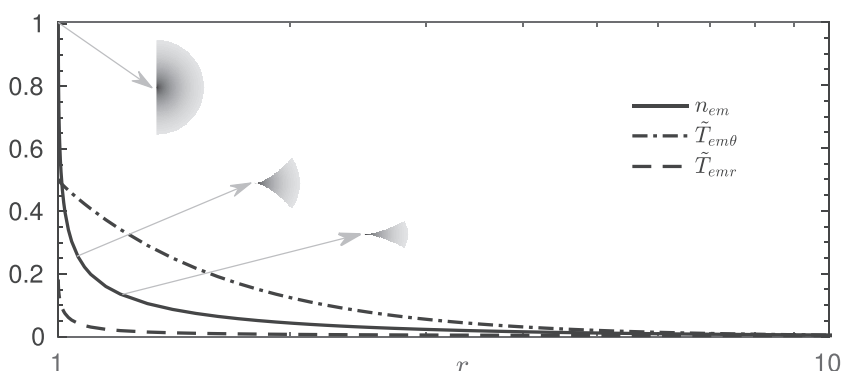

FIG. 6. The density profile, kinetic temperature profile, and the directional distribution $\left(\epsilon_{e m} \leq 2\right)$ of emitted electrons for $\rho_{0}=1, \delta_{i}=1, \delta_{e m}=0.34$, $\beta=11.81$, and $\varphi_{p}=-40$.

obtained by scaling and offsetting those for plasma electrons. For the $\tilde{u} r$-diagram, scaling and offsetting of the $y$-axis are applied to Fig. 5(a) as

$$
\tilde{u}_{e m r}\left(r, l^{2}\right)=\tilde{u}_{e r}\left(r, \delta_{\mathrm{em}} l^{2}\right) / \delta_{e m}+\varphi_{p} / \delta_{e m} .
$$

The $\epsilon_{e}=40$ and $\epsilon_{e}=0$ lines thus become $\epsilon_{e m}=0$ and $\epsilon_{e m}=-117.65$, respectively. As electrons move outwards, all $\tilde{u}_{\text {emr }}$ curves decrease monotonically, suggesting that all emitted electrons are accelerated towards infinity. For the $\epsilon l$ diagram, both axes are scaled/offset as

$$
\begin{gathered}
\epsilon_{e m}=\left(\epsilon_{e}+\varphi_{p}\right) / \delta_{e m} \\
l_{e m r}^{2}\left(r, \epsilon_{e m}\right)=r^{2}\left(\epsilon_{e}-u_{e r}\right) / \delta_{e m}=l_{e r}^{2}\left(r, \epsilon_{e}\right) / \delta_{e m} .
\end{gathered}
$$

The first quadrant then corresponds to the top-right area enclosed by the two dotted lines in Fig. 5(b), and the envelope is still outside the first quadrant as that for plasma electrons. However, different from plasma electrons, the orbits of emitted electrons are followed from the probe outwards. Therefore, the $l_{e m r}^{2}$ lines always move to the right in the first quadrant due to the decreasing slope. At the end, all the electrons that are emitted at the probe are non-forbidden at any radius as $l_{e m r}^{*}=l_{e m R}$ for all $\epsilon_{e m} \geq 0$, congruent with that observed in the $\tilde{u} r$-diagram.
For the distribution functions in Fig. 6, the HM electrons populate the entire $\epsilon_{r}>0$ plane at the probe. With no electrons reflected back, the $\epsilon_{r}<0$ plane is unpopulated-not only at the probe but also at any other $r$. In the $\epsilon_{r}>0$ plane for $r>1$, only one type of holes due to absorption radius appears for all energies $\epsilon_{e m}>0$, crossing the $\epsilon_{\theta}$-axis and limited by $\theta_{1}$. The absorption radius for any energy is at the probe as $l_{e m r}^{*}=l_{e m R}$. Since $\theta_{1}=\arcsin \left(l_{\text {emR }} / l_{\text {emr }}\right)$ decreases with $r$, the hole grows bigger and the density drops. With all emitted electrons accelerated outwards and present everywhere, the azimuthal kinetic temperature $\tilde{T}_{e m \theta}$ in Fig. 6 can only drop with decreasing $\left|v_{\theta}\right|$, instead of being nonmonotonic as $\tilde{T}_{e \theta}$ in Fig. 5(c). In the radial direction, $\tilde{T}_{e m r}$ also drops with $r$, seeing that a reduced dispersion is observed as all $\tilde{u}_{e m r}$ curves come together at infinity in Fig. 5(a).

\section{NON-MONOTONIC POTENTIAL, $\varphi_{p}<0$}

For $\rho_{0}=1, \delta_{i}=1, \delta_{\text {em }}=0.32, \beta=1.72$, and $\varphi_{p}=-0.5$, the potential profile is non-monotonic (operational regime 5 in Ref. 21), with a minimum $\varphi_{m}=-0.565$ at $r_{m}=1.41$. However, both the extreme curve $\tilde{u}_{\alpha m}$ in the $\tilde{u} r$-diagram and the envelope in the $\epsilon l$-diagram are monotonic.

\section{A. Plasma ions}

Although the potential is not monotonic, the ion collection mechanism is interestingly simple in this case. First of all, because of the negative potential energy $u_{i r}<0$, the nonmonotonic potential does not introduce electric potential barriers for ions. Then, in the $\epsilon$-diagram in Fig. 7(b), with the envelope outside the first quadrant, the $l_{i r}^{2}$ line always moves to the left there as ions move inwards. Therefore, the nonforbidden condition is sufficient, with no absorption radius for any $\epsilon_{i}$ at any $r\left(l_{i r}^{*}=l_{i r}\right.$ for all $\left.\epsilon_{i}>0\right)$. The ion collection is thus OML as $l_{i}^{*}=l_{i R}$ for all energies.

For the distribution functions in Fig. 7(c), there is only the type of holes due to captured ions. These holes appear
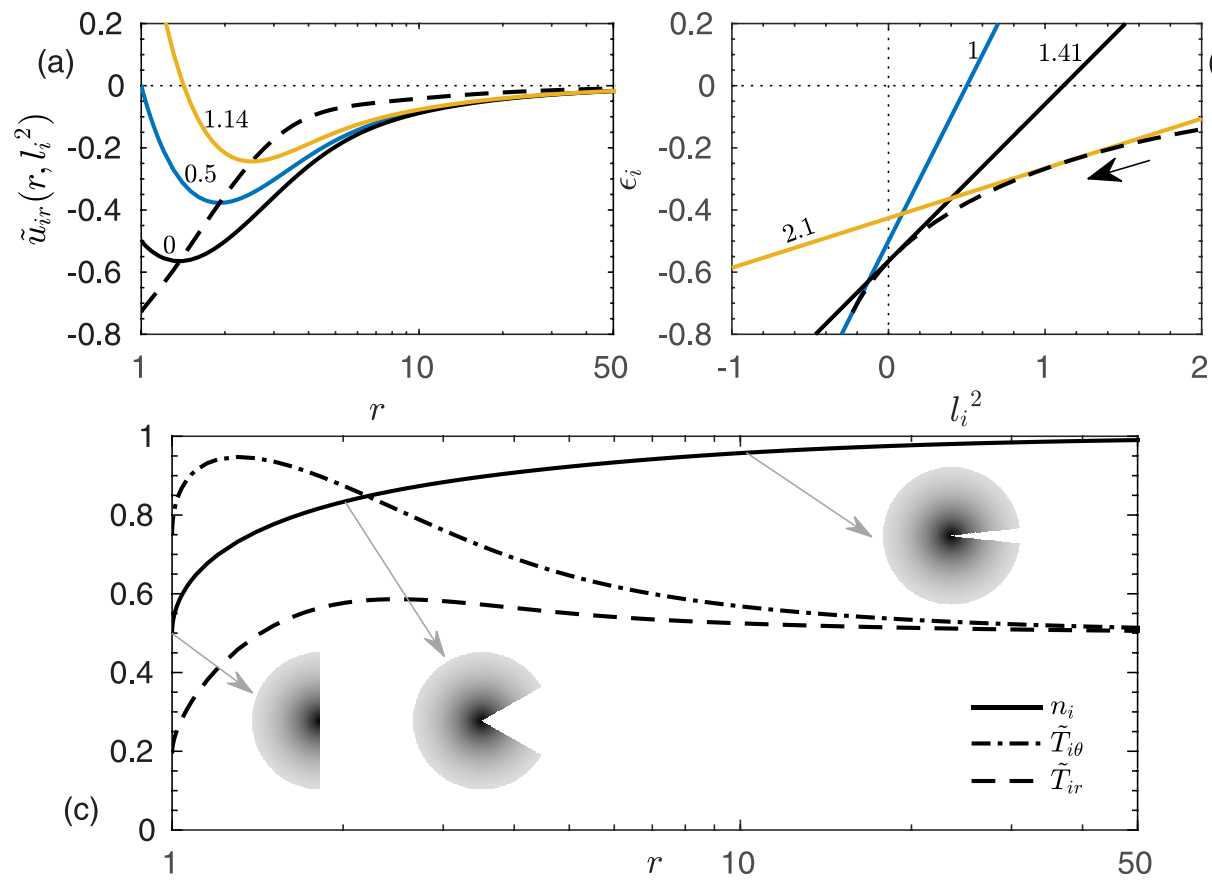
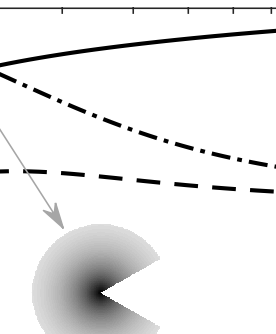
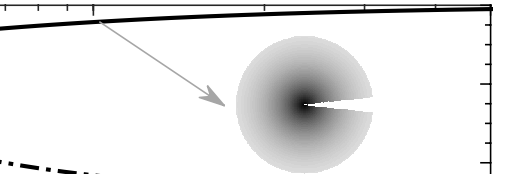

(b)

FIG. 7. For $\rho_{0}=1, \delta_{i}=1, \delta_{\text {em }}=0.32$, $\beta=1.72, \varphi_{p}=-0.5$, and plasma ions: (a) ür-diagram; (b) $\epsilon$-diagram; (c) density profile, kinetic temperature profile, and the directional-energy distribution $\left(\epsilon_{i} \leq 2\right)$. 
for all $\epsilon_{i}>0$ and cross the $\epsilon_{r}$-axis. The $\theta_{0}=\arcsin \left(l_{e R} / l_{e r}\right)$ limit increases with reducing $r$. The density in Fig. 7(c) thus drops monotonically until being half at the probe, congruent with the OML condition.

To discuss the kinetic temperatures in Fig. 7(c), we need to again use the $\tilde{u} r$-diagram in Fig. 7(a). At infinity, given by Eqs. (31a) and (29), the effective potential $\tilde{u}_{i r}$ curves lie below the extreme curve $\tilde{u}_{i m}$ due to $r \mathrm{~d} u_{i r} / \mathrm{d} r \sim 1 / r>l_{i}^{2} / r^{2}$. So, the $\tilde{u}_{i r}$ curves start below $\tilde{u}_{i m}$ at infinity and drop until crossing $\tilde{u}_{i m}$ at their minima. Due to these minima, as those observed for $1.16<r<7.47$ in Fig. 4(a), the radial kinetic temperature $\tilde{T}_{i r}$ rises at further $r$ locations due to increasing dispersion whereas drops near the probe. The minima here have a lowest value $\left(\tilde{u}_{i}=-0.565\right)$ that is much higher than that in Fig. 4(a) $\left(\tilde{u}_{i} \approx-16\right)$, yielding a $\tilde{T}_{i r}$ smaller than that in Fig. 4(c). In the azimuthal direction, $\tilde{T}_{i \theta}$ increases at further $r$ due to angular momentum conservation and decreases near the probe because of the loss of high- $l_{i}$ ions owing to orbital effects. The reason for the $\tilde{T}_{i \theta}$ to be smaller than that in Fig. 4(c) can be explained by the differences in $\epsilon l$-diagrams. The region to the right of the $l_{i r}^{2}$ lines represents a loss of high- $l_{i}$ ions that limits the increase of $\tilde{T}_{i \theta}$. Shown by Fig. 7(b), this loss appears for $l_{i}^{2} \gtrsim 0.5$ in this case, a lost-region much larger than that in Fig. 4(b) $\left(l_{i}^{2} \gtrsim 40\right)$.

Compared to Sec. IV A, although ion collection is OML in both cases $\left(l_{i}^{*}=l_{i R}\right)$, the envelope is outside the first quadrant only in this case and the orbital barrier is always set by the local conditions at $r\left(l_{i r}^{*}=l_{i r}\right)$. The density is thus monotonic in Fig. 7(c) but not in Fig. 4(c) as $l_{i r}^{*}=l_{i r}$ is no longer satisfied at every $r$.

\section{B. Plasma electrons}

The positive potential energy for plasma electrons $\left(u_{e r}>0\right)$ results in electric potential barriers with the maximum $u_{m}=0.565$ at $r_{m}=1.41$. Electrons are "repelled" for $r>r_{m}$, yet "attracted" for $r<r_{m}$.
For $r>1.41$, the envelope in Fig. 8(b) is outside the first quadrant and meets the positive $\epsilon_{e}$-axis at $r=1.41$. In this $r$ region, plasma electrons behave similar to that described in Sec. IV B. Only electrons with energy above the electric potential barrier $\left(\epsilon_{e}>u_{e r}^{*}=u_{e r}\right)$ are allowed at $r$. For any of these sufficient energies, the non-forbidden condition is sufficient as $l_{e r}^{*}=l_{e r}$ for $\epsilon_{e}>u_{e r}$. The two kinds of holes in distribution functions can now be seen in Fig. 8(c). The centred hole for $\epsilon_{e}<u_{e r}$ is due to electric potential barriers. The other hole due to the captured electrons appears for $\epsilon_{e}>u_{e}^{*}=u_{m}$, which is different from $\epsilon_{e}>u_{e R}$ in Sec. IV B because the electric potential barrier for electrons to arrive at the probe is set by $u_{m}$ at $r_{m}$, instead of $u_{e R}$ as $u_{m}>u_{e R}$. Since $u_{e r}$ and $\theta_{0}=\arcsin \left(l_{e}^{*} / l_{e r}\right)$ increase, both holes grow bigger and the density drops with decreasing $r$.

For $1<r<1.41$, the electric potential barrier does not change with $r$ as $u_{e r}^{*}=u_{m}$. The envelope in the $\epsilon$-diagram in Fig. 8(b) now enters the first quadrant. For $u_{m}<\epsilon_{e}$ $<\epsilon_{e, \text { env }}(r)$, the angular momentum for electrons to arrive at $r$ is limited by the envelope as $l_{e r}^{*}=l_{e, e n v}\left(r_{\epsilon}^{*}\right)$. For $\epsilon_{e}>u_{e, \text { env }}(r)$, there is no absorption radius as $l_{e r}^{*}=l_{e r}$. The condition for electrons to be captured by the probe is thus given by $l_{e}^{*}=l_{e, e n v}\left(r_{\epsilon}^{*}\right)$ for $u_{m}<\epsilon_{e}<\epsilon_{e, e n v}(R)$ and $l_{e}^{*}=l_{e R}$ for $\epsilon_{e}>\epsilon_{e, e n v}(R)$. For the distribution functions in Fig. 8(c), all three kinds of holes appear. The centred hole due to the electric potential barrier does not vary in this $r$ region due to $u_{e r}^{*}=u_{m}$. For $u_{m}<\epsilon_{e}<u_{e, e n v}(r)$, the other two types of holes-due to absorption radius and captured electronsappear as one single hole limited by $\theta_{1}$ in the $\epsilon_{r}<0$ plane, for the reason that they are joined in the $\epsilon_{r}>0$ plane due to $\theta_{1}=\theta_{0}=\arcsin \left[l_{e, e n v}\left(r_{\epsilon}^{*}\right) / l_{e r}\right]$. As electrons move inwards, such a hole grows bigger since $\theta_{1}$ decreases with increasing $l_{e r}$ [see Fig. 8(b)]. For $\epsilon_{e}>u_{e, e n v}(r)$, only the captured-electron hole limited by $\theta_{0}=\arcsin \left[l_{e}^{*} / l_{e r}\right]$ appears, which grows due to decreasing $l_{e r}$. Since all holes grow bigger, the density drops for $1<r<1.41$ yet at a lower rate compared to the
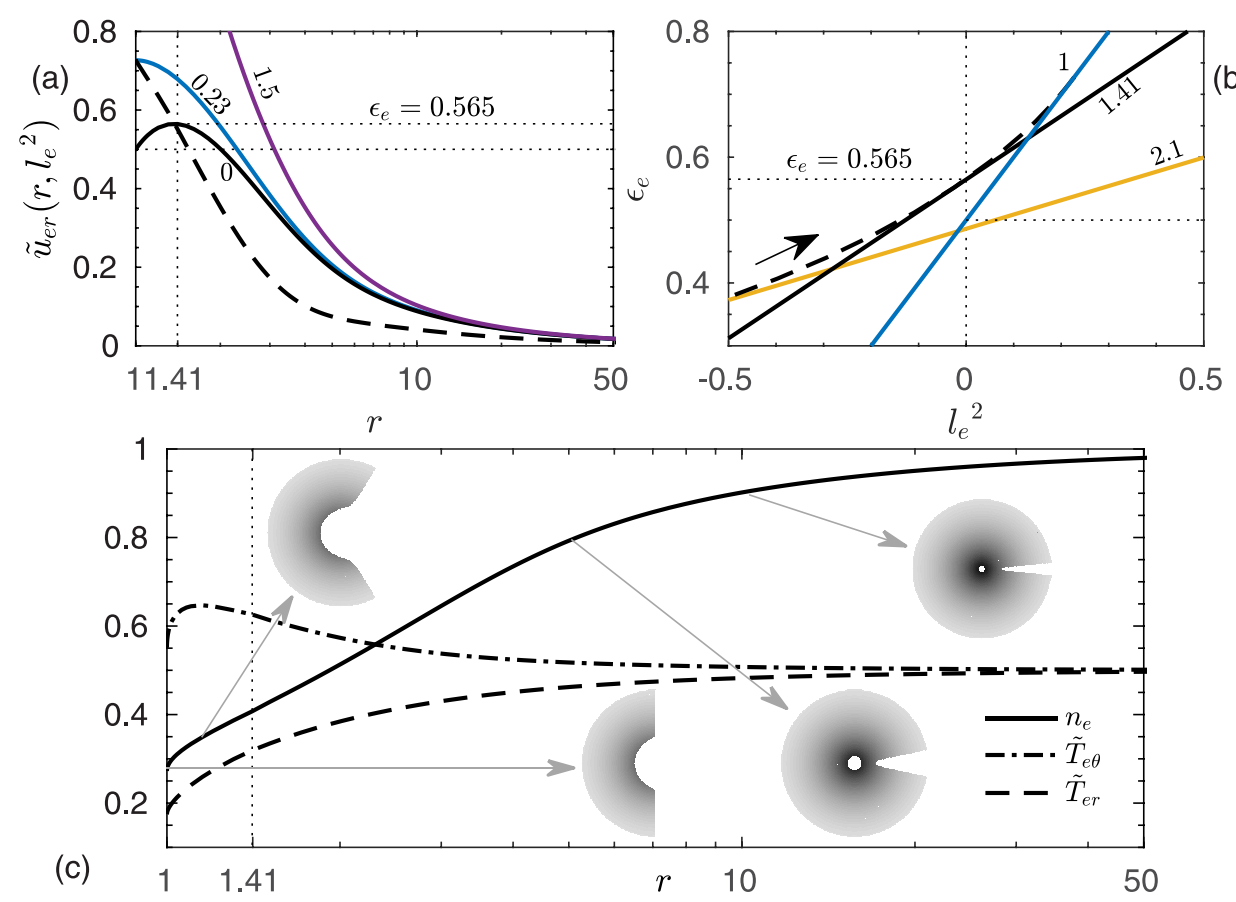

(c)

$1 \quad 1.41$

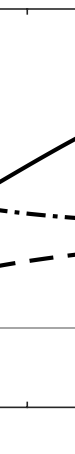

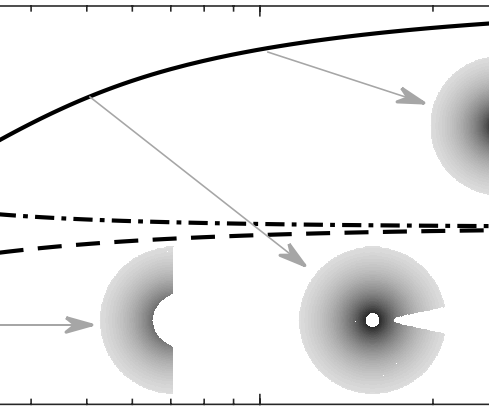

0.5 (b)

FIG. 8. For $\rho_{0}=1, \delta_{i}=1, \delta_{\text {em }}=0.32$, $\beta=1.72, \varphi_{p}=-0.5$, and plasma electrons: (a) $\tilde{u} r$-diagram; (b) $\epsilon l$-diagram; (c) density profile, kinetic temperature profile, and the directional distribution $\left(\epsilon_{e} \leq 2\right)$. 
other $r$ region due to the invariant size of the centred hole with high- $f_{\alpha}$ values.

The kinetic temperature in Fig. 8(c) describes a similar behaviour to that shown in Fig. 5(c). A comparatively higher $\tilde{T}_{e \theta}$ in this case can be explained by the reduced electric potential barrier that allows more increase in $\left|v_{\theta}\right|$.

\section{Emitted electrons}

Due to the electric potential hill inside the virtual cathode, emitted electrons are "repelled" by the electric field until the maximum potential energy $u_{m}=0.203$ at $r_{m}=1.14$, beyond which they are accelerated all the way to infinity. The distribution functions in Fig. 9, being more complicated than in Fig. 6, require studying first the $\epsilon$-diagram for all $r$ locations.

The $\epsilon l$-diagram for emitted electrons is again obtained by scaling and offsetting Fig. 8(b), in which the first quadrant corresponds to the top-right region enclosed by the dotted lines. At $r=1$, the electrons that are emitted by the probe occupy the region to the left of the $l_{\text {emR }}^{2}$ line, which is tangent to the envelope at $\epsilon_{e m}=\epsilon_{\text {em,env }}(R)$. For $1<r<1.41$, the $l_{e m r}^{2}$ line crosses the positive $\epsilon_{e m}$-axis at $u_{e m r}$ and osculates the envelope at $\epsilon_{e m}=\epsilon_{e m, e n v}(r)$. The electrons that do not have enough energy to overcome the electric potential hill $\left(\epsilon_{e m}<u_{e m r}\right)$ are reflected back to the probe from $r$. For $u_{e m r}<\epsilon_{e m}<\epsilon_{\text {em,env }}(r)$, the non-forbidden condition is sufficient $\left(l_{\text {emr }}^{*}=l_{\text {emr }}\right)$ because the $l_{e m r}^{2}$ line lies to the left of any $l_{e m r^{\prime}}^{2}$ line for $r^{\prime}<r$. For $\epsilon_{e m, e n v}(r)<\epsilon_{e m}<\epsilon_{e m, e n v}(R)$, the $l_{e m r}^{*}$ boundary is given by the envelope as $l_{e m r}^{*}=l_{e m, e n v}\left(r_{\epsilon}^{*}\right)$ and the absorption radius $r_{\epsilon}^{*}$ is somewhere between the probe and that $r$ location. For $\epsilon_{e m}>\epsilon_{\text {em,env }}(R)$, the absorption radius is at the probe as $l_{e m r}^{*}=l_{e m R}$. At $r_{m}=1.41$, the $l_{e m r}^{2}$ line is tangent to the envelope at its intersection with the positive $\epsilon_{e m}$-axis as $\epsilon_{e m}=u_{m}$. The electrons that can escape the virtual cathode correspond to the region to the left of the envelope for $u_{m}<\epsilon_{e m}<\epsilon_{\text {em,env }}(R)$ as $l_{e m}^{*}=l_{\text {em,env }}\left(r_{\epsilon}^{*}\right)$ and the $l_{e m R}^{2}$ line for $\epsilon_{e m}>\epsilon_{e m, e n v}(R)$ as $l_{e m}^{*}=l_{e m R}$. Beyond $r_{m}$, the envelope in Fig. 8(b) is outside the first quadrant and the $l_{\text {emr }}^{2}$ lines move always to the right in that quadrant. So, there is no any barrier for the electrons that have passed the electric potential hill to be captured by the plasma at infinity.

For the distribution function at the probe [see Fig. 9], it is HM in the $\epsilon_{r}>0$ plane (the same as Fig. 6). In the $\epsilon_{r}<0$ plane, some region is populated by the electrons that are reflected back to the probe (different from Fig. 6). As explained above, for $\epsilon_{e m}<u_{m}$, all emitted electrons are reflected back by the electric potential hill, thus occupying

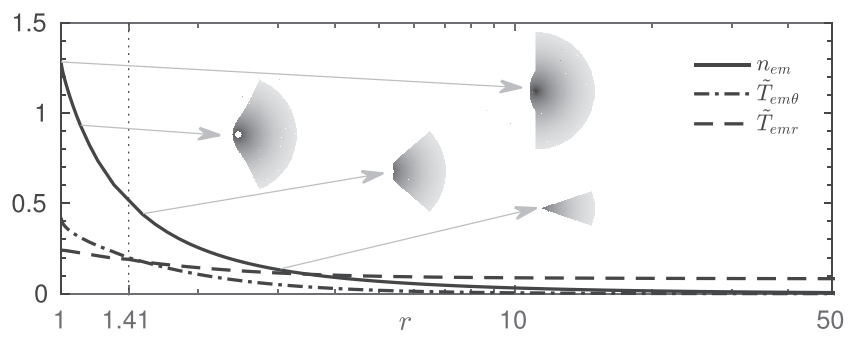

FIG. 9. The density profile, kinetic temperature profile, and the directional distribution $\left(\epsilon_{e m} \leq 2\right)$ of emitted electrons for $\rho_{0}=1, \delta_{i}=1, \delta_{e m}=0.32$, $\beta=1.72$, and $\varphi_{p}=-0.5$. the entire half circle as $\theta_{0}=0$. For $u_{m}<\epsilon_{\text {em }}<\epsilon_{\text {em,env }}(R)$, electrons are reflected back by orbital effects with constraints on angular momentum as $l_{e m}^{*}=l_{\text {em,env }}\left(r_{\epsilon}^{*}\right)$, thus populating the $\epsilon_{r}<0$ plane for $\pi / 2>\theta>\theta_{0}=\arcsin \left(l_{\text {em,env }} / l_{\text {emR }}\right)$. The electrons emitted with higher energies $\epsilon_{\text {em }}>\epsilon_{\text {em,env }}(R)$ do not come back due to $l_{e m}^{*}=l_{e m R}$, yielding $\theta_{0}=\pi / 2$. So, the kind of hole that appears here is the one bound by the captured boundary $l_{e m}^{*}$. At the end, the density at the probe is above one due to these reflected electrons.

At any $r$ inside the virtual cathode $(1<r<1.41)$, the distribution vanishes for $\epsilon_{e m}<u_{e m r}$. For $u_{e m r}<\epsilon_{e m}<u_{m}$, in the $\epsilon_{r}>0$ plane for outgoing electrons, entire half circles are covered due to $l_{e m r}^{*}=l_{e m r}$ and $\theta_{1}=\pi / 2$. Because all these outgoing electrons cannot pass the electric potential hill at $r_{m}$, they all come back to $r$ as incoming electrons and cover also the half circles in the $\epsilon_{r}<0$ plane. For $u_{m}<\epsilon_{e m}$ $<\epsilon_{e m, e n v}(r)$, the condition $l_{e m r}^{*}=l_{e m r}$ again results in the half circles fully covered in the $\epsilon_{r}>0$ plane. Nevertheless, in the $\epsilon_{r}<0$ plane, due to $l_{e m}^{*}<l_{e m r}$, some electrons come back to $r$ and cover an azimuthal angle range $\arcsin \left(l_{e m}^{*} / l_{\text {emr }}\right)=\theta_{0}<\theta<\pi / 2$. For $\epsilon_{\text {em }}>\epsilon_{\text {em,env }}(r)$, the electrons that fulfill the sufficient condition $l_{e m} \leq l_{e m r}^{*}$ can arrive at $r$, without being reflected back owing to $l_{e m r}^{*}=l_{e m}^{*}$. These electrons only populate the $\epsilon_{r}>0$ plane for a range $0<\theta<\theta_{1}=\arcsin \left(l_{e m}^{*} / l_{e m r}\right)$. So, all three kinds of holes appear here. They all grow bigger with $r$ because $u_{e m r}$ rises, $\theta_{0}$ for $u_{m}<\epsilon_{e m}<\epsilon_{e m, e n v}(r)$ increases, and $\theta_{1}$ for $\epsilon_{e m}$ $>\epsilon_{\text {em,env }}(r)$ decreases. The density thus drops.

For $r>1.41$, there are only the electrons that escape the virtual cathode and populate the $\epsilon_{r}>0$ plane of distribution functions. These electrons have energies as $\epsilon_{e m}>u_{m}$. Due to the overlapped sufficient and captured boundaries $\left(l_{e m}^{*}=l_{e m r}^{*}\right)$, such a populated region is bound by $\theta_{1}=\theta_{0}=\arcsin \left(l_{e m}^{*} / l_{\text {emr }}\right)$. Slight differences are found in the two distribution functions presented for this $r$-range. For the one at larger $r$, the condition $\theta_{0}=0$ at $\epsilon_{e m}=u_{m}$ can be clearly observed. For the other one, this condition still holds. Nevertheless, because $l_{e m r}$ is now comparable with $l_{e m}^{*}$, the sharp decline of $l_{e m}^{*}$ close to $\epsilon_{e m}=u_{m}$ [see the envelope in Fig. 8(b)] is more pronounced here as a sudden drop in $\theta_{0}$ in the distribution function. Eventually, the density continues dropping with $r$ in this region due to decreasing $\theta_{0}$, yet at a lower rate.

The kinetic temperatures, $\tilde{T}_{e m r}$ and $\tilde{T}_{e m \theta}$, also drop with $r$. Compared with Fig. 6, a higher $\tilde{T}_{e m r}$ is clearly observed. Due to the electric potential hill, emitted electrons are no longer always accelerated in the radial direction. Some electrons with angular momentum close to zero are actually deaccelerated inside the virtual cathode as seen in Fig. 8(a). This phenomenon, which does not appear for the monotonic potential profile [see Fig. 5(a)], gives an explanation for more dispersion in $v_{r}$.

\section{NON-MONOTONIC POTENTIAL, $\varphi_{p}>0$}

For $\rho_{0}=1, \delta_{i}=1, \delta_{e m}=0.32, \beta=1.72, \quad$ and $\varphi_{p}$ $=0.1$, the potential profile is non-monotonic at a positive probe bias (operational regime 4 in Ref. 21). There is a potential minimum $\varphi_{m}=-0.194$ at $r_{m}=2.12$. The kinetic features for this case are actually very similar to those 
discussed in Sec. V due to the monotonic envelope. So, they will be briefly discussed in this section, emphasizing more the differences. In this work, we have chosen to present a case with the monotonic envelope. Nevertheless, as observed in numerical results not shown here, the envelope can be non-monotonic in some cases.

In ion distribution functions [see Fig. 10(c)], different from Fig. 7(c) with only the hole for captured ions, a centred hole due to electric potential barriers appears for $1<r<1.12$. The distributions vanish in this hole because the potential energy $u_{i r}$ is larger than that at infinity $\left(u_{i r}>0\right)$ [see Fig. 10(a)]. The ions captured by the probe thus must have energies $\epsilon_{i}>u_{i p}$. Therefore, the other hole due to captured ions exists only for $\epsilon_{i}>u_{i p}$ (but at all $r$ ). The hole bound by the sufficient boundary $l_{i r}^{*}$ does not appear, for the reason that the envelope in Fig. 10(b) lies below the first quadrant, giving $l_{i r}^{*}=l_{i r}$ for any positive energy. Since the $l_{i r}^{2}$ line always moves to the left as ions move inwards, this hole grows bigger all the way to the probe with increasing $\theta_{0}=\arcsin \left(l_{i}^{*} / l_{i r}\right)$. The density thus drops towards the probe, yet more rapid for $1<r<1.12$ due to the loss of high- $f_{i}$ ions. Compared to Fig. 7(c), the radial kinetic temperature $\tilde{T}_{i r}$ is close to each other in both cases, yet $\tilde{T}_{i \theta}$ is smaller in this case. This can be again explained by looking at the differences in $\epsilon l$-diagrams, where the lost-region of high- $l_{i}$ ions is clearly larger in Fig. 10(b).

For plasma electrons, compared to Sec. V B, the difference is that the potential energy in Fig. 11(a) becomes smaller than zero for $1<r<1.12$. Nevertheless, this difference does not lead to distinct behaviours in kinetic features, in the reason that the electric potential barrier for $1<r<r_{m}$ is always set by the maximum potential energy at $r_{m}$, irrelevant to the drop of potential energy inside the virtual cathode. In this case, this maximum potential energy $u_{m}$ is lower $(0.194<0.565)$ but with more drop inside the virtual cathode $(0.294>0.065)$, leading to a higher density in Fig. 11 (c). In addition, the reduced $u_{m}$ also gives rise to smaller lost-regions for high- $l_{e}$ electrons in Fig. 11(b) and thus a higher $\tilde{T}_{e \theta}$ in Fig. 11(c).

For emitted electrons, the maximum potential energy $u_{m}=0.919$ occurs at $r_{m}=2.12$. Compared to Sec. V C, more electric potential barrier leads to a larger centred hole in distribution functions and a more rapid density drop inside the virtual cathode [see Fig. 12]. The kinetic temperatures in both directions are very close to those in Fig. 9, with $\tilde{T}_{e m r}$ being slightly higher in Fig. 12.

\section{CONCLUSIONS}

Based on the OMT, a kinetic model for cylindrical emitters is constructed and the Vlasov-Poisson system is solved self-consistently as long as collisions, plasma drift, particle trapping, transient effects, and magnetic fields are not significant. Once the potential profile $\varphi(r)$ is found, the kinetic features-orbital motions, local distributions, and macroscopic quantities - are disclosed.

In the plane of two orbital invariants $\left(\epsilon_{\alpha}\right.$ versus $\left.l_{\alpha}^{2}\right)$, orbital motions are distinguished by three boundaries, nonforbidden boundary $l_{\alpha r}$, sufficient boundary $l_{\alpha r}^{*}$, and captured boundary $l_{\alpha}^{*}$, together with their energy preconditions ( $u_{\alpha r}, u_{\alpha r}^{*}$, and $u_{\alpha}^{*}$, respectively). The $l_{\alpha r}^{*}$ and $l_{\alpha}^{*}$ boundaries that bound the domains for particles to exist at $r$ [see Eq. (10)] can be found by constructing an $r$-family of $l_{i r}^{2}$ lines in the $\epsilon l$ diagram. The $r$-parametrized envelope of these lines reveals distinct orbital motions in $r$ regions that are separated by critical $r$ locations such as corners or intercepts of the envelope.

By constructing an $l$-family of effective potential $\tilde{u}_{\alpha r}$ curves, the change of radial velocity $v_{r}$ along an orbit with $\epsilon_{\alpha}$ and $l_{\alpha}$ is given by the distance from the correspondent $\tilde{u}_{\alpha r}$ curve to the horizontal $\epsilon_{\alpha}$ line and the particle is reflected at their intersection, which thus illustrates again the constraints on these two orbital invariants. The cross of the $\epsilon_{\alpha}$ line and the extreme curve $\tilde{u}_{\alpha m}$ can also give the absorption radius $r_{\epsilon}^{*}$ for $\epsilon_{\alpha}$. Most importantly, this $\tilde{u} r$-diagram shows the dispersion in $v_{r}$.
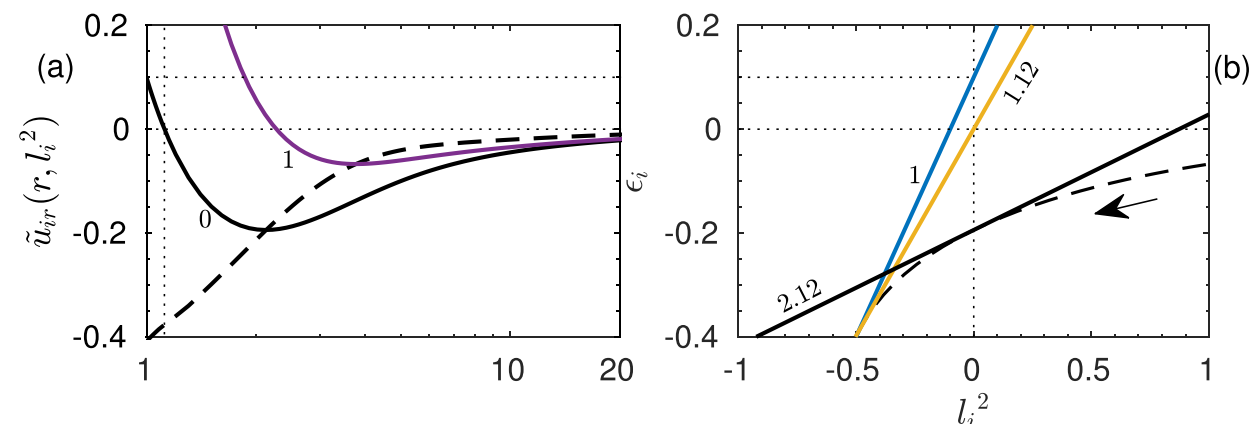

(c)

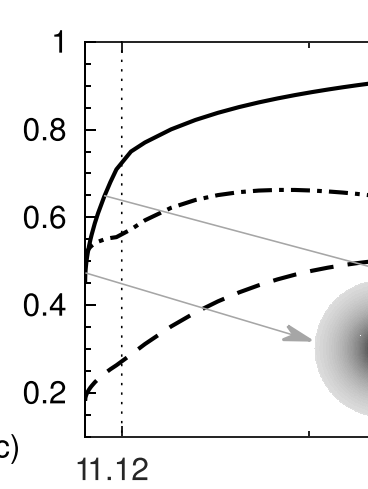

11.12

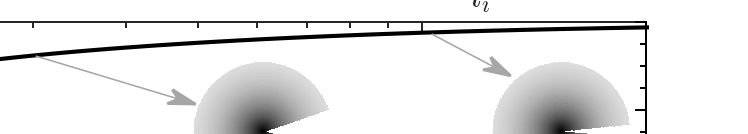

9
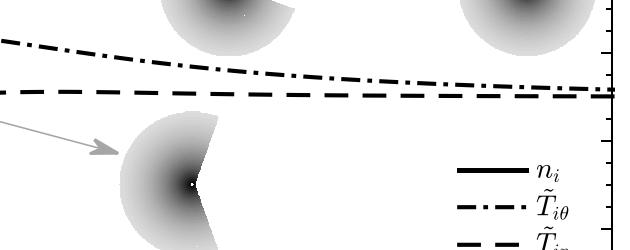

$\longrightarrow n_{i}$

$-\cdot \cdot \tilde{T}_{i}$

$-\tilde{T}_{i r}$
FIG. 10. For $\rho_{0}=1, \delta_{i}=1, \delta_{\text {em }}=0.32$, $\beta=1.72, \varphi_{p}=0.1$, and plasma ions: (a) $\tilde{u} z$-diagram; (b) $\epsilon$-diagram; (c) density profile, kinetic temperature profile, and the directional-energy distribution $\left(\epsilon_{i} \leq 2\right)$. 


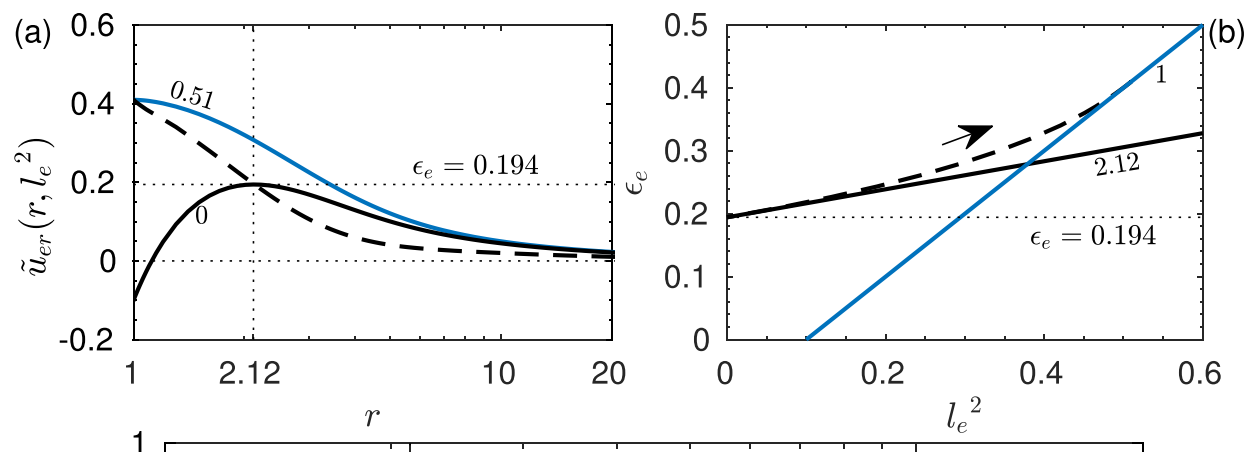

(c)

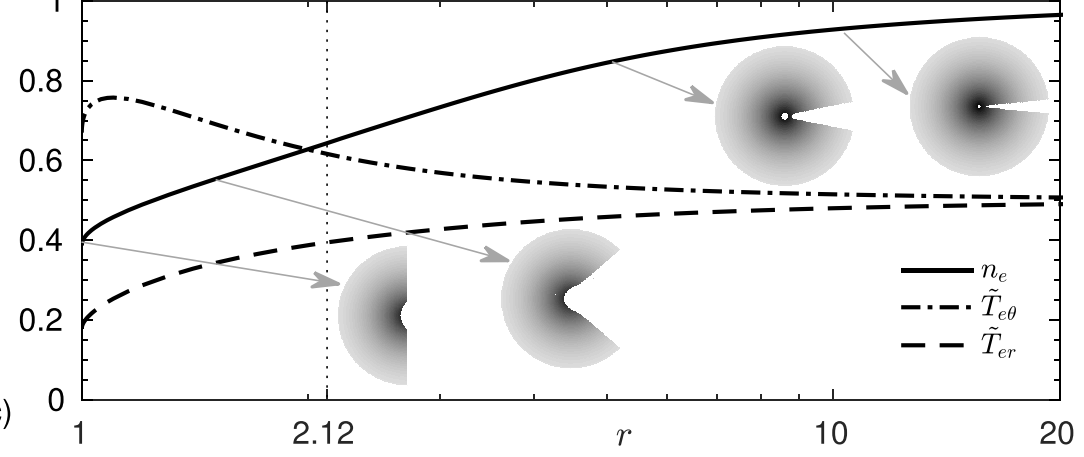

FIG. 11. For $\rho_{0}=1, \delta_{i}=1, \delta_{e m}=0.32$, $\beta=1.72, \varphi_{p}=0.1$, and plasma electrons: (a) $\tilde{u} r$-diagram; (b) $\epsilon l$-diagram; (c) density profile, kinetic temperature profile, and the directional-energy distribution $\left(\epsilon_{e} \leq 2\right)$
The local distribution functions have been presented versus a directional energy $\epsilon_{r} \epsilon_{\theta}$-plane with the radial coordinate identical to $\epsilon_{\alpha}$. The distribution $f_{\alpha}$ (if not zero) is not only isotropic for any azimuthal coordinate $\theta$ in this $\epsilon_{r} \epsilon_{\theta}$-plane but also invariant for different $r$ locations. The holes in distributions can only be bound by three kinds of boundaries - the sufficient boundary $l_{\alpha r}^{*}\left(\theta_{1}\right)$, the captured boundary $l_{\alpha}^{*}\left(\theta_{0}\right)$, and the electric potential barrier $u_{\alpha r}^{*}$. The variation of these boundaries with $r$ is intelligibly given by the $\epsilon l$-diagram. The variation of particle densities can then be discussed by looking at the change of populated regions in distributions.

Together with the macroscopic quantities, the orbital invariant $\epsilon$-diagram, the effective potential $\tilde{u} r$-diagram, and the distribution function $\epsilon_{r} \epsilon_{\theta}$-diagram have been presented in this work using the quantitative results calculated numerically. By following the procedure described above, the kinetic features are unfold. The density variation can be discerned from the $\epsilon_{r} \epsilon_{\theta}$-diagram. The radial kinetic temperature $\tilde{T}_{\alpha r}$ can be related to the dispersion of $v_{r}$ shown in the $\tilde{u} r$-diagram. In the azimuthal direction, $\tilde{T}_{\alpha \theta}$ tends to increase towards the probe due to conservation of angular momentum. Nevertheless, orbital effects exclude high- $l_{\alpha}$ particles and thus limit such an increase. This phenomenon can be explained by looking at the lost region for these high- $l_{\alpha}$ particles in the $\epsilon l$-diagram. From the kinetic temperatures, it is clearly seen that a fluid model is

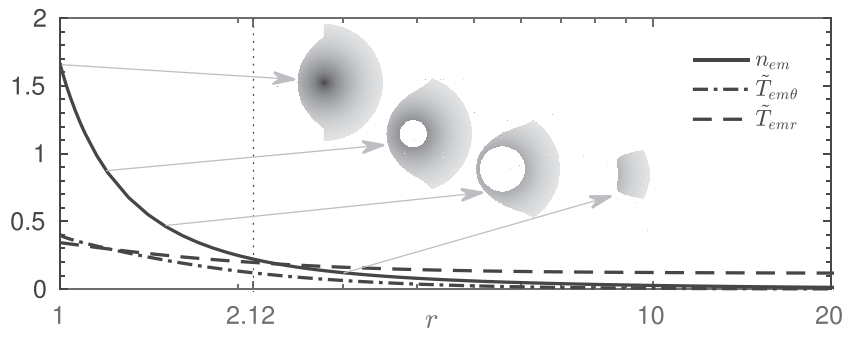

FIG. 12. For $\rho_{0}=1, \delta_{i}=1, \delta_{e m}=0.32, \beta=1.72$, and $\varphi_{p}=0.1$, the density profile, kinetic temperature profile, and the directional-energy distribution functions $\left(\epsilon_{e m} \leq 2\right)$. only adequate for emitted electrons in the case of monotonic potential and negative probe bias.

The importance of the envelope in the $\epsilon l$-diagram has been highlighted in this work as a groundwork for kinetic features. On top of this, this envelope can provide a guideline for analytical analyses. ${ }^{13,15,19}$ Moreover, the resolution of the envelope can serve as one of the criteria to refine the $r$ mesh used in numerical calculations. Certainly, as a Vlasov-Poisson solver, the $r$ mesh should be fine enough to have good resolutions on the density and the potential profile. In addition, since the envelope can determine the sufficient boundary as $l_{\alpha r}^{*}$ $=l_{\alpha, e n v}\left(r_{\epsilon}^{*}\right)$ and yet the values can only be evaluated at $r_{\epsilon}^{*}$ that has been defined at the mesh, the resultant envelope must have a sufficient resolution in $\epsilon_{\alpha, \text { env }}(r)$ and $l_{\alpha, \text { env }}(r)$.

Some hypotheses in this work can be easily relaxed within the framework of the model. For Maxwellian plasma and HM emitted electrons, any energy distribution (e.g., Druyvesteyn distribution) can be substituted into Eq. (8) that is later used in the integro-differential equation for the Vlasov-Poisson system. For trapped particles, with their $\epsilon l$ domain pointed out in Sec. II B, the model can be extended if a law for the distribution function inside this trapped domain is prescribed (as long as the electric field is symmetric). It is also straightforward to include multiple plasma or emitted species. The relaxation of other hypotheses (collisions, flowing plasmas, probe end effects, etc.), which can violate the conservations of distribution function, energy, and angular momentum, would require computationally expensive algorithms. ${ }^{25}$ However, the theory presented in this work can be used as a benchmark case to validate more complex codes.

\section{ACKNOWLEDGMENTS}

This work was supported by the Ministerio de Economía y Competitividad of Spain (Grant No ESP 2016-75887). Work by G. Sánchez-Arriaga was supported by the Ministerio de Economía y Competitividad of Spain (Grant No. RYC-2014-15357). 
${ }^{1}$ J. P. Sheehan and N. Hershkowitz, Plasma Sources Sci. Technol. 20, 063001 (2011).

${ }^{2}$ J. P. Sheehan, Y. Raitses, N. Hershkowitz, and M. McDonald, J. Propul. Power 33, 614 (2017).

${ }^{3}$ J. Cavalier, N. Lemoine, G. Bousselin, N. Plihon, and J. Ledig, Phys. Plasmas 24, 013506 (2017)

${ }^{4}$ G. L. Delzanno and X. Z. Tang, Phys. Rev. Lett. 113, 035002 (2014).

${ }^{5}$ X. Wang, J. Pilewskie, H.-W. Hsu, and M. Horányi, Geophys. Res. Lett. 43, 525, doi:10.1002/2015GL067175 (2016).

${ }^{6}$ B. Thiebault, A. Hilgers, E. Sasot, H. Laakso, P. Escoubet, V. Genot, and J. Forest, J. Geophys. Res. A 109, A12207 (2004).

${ }^{7}$ J. D. Williams, J. R. Sanmartin, and L. P. Rand, IEEE Trans. Plasma Sci. 40, 1441 (2012).

${ }^{8}$ G. Sanchez-Arriaga and X. Chen, "Modeling and performance of electrodynamic low-work-function tethers with photoemission effects" (published online).

${ }^{9}$ M. Martínez-Sánchez and E. Ahedo, Phys. Plasmas 18, 033509 (2011).

${ }^{10}$ M. Martínez-Sánchez, J. Navarro-Cavallé, and E. Ahedo, Phys. Plasmas 22, 053501 (2015).

${ }^{11}$ M. W. Liemohn and G. V. Khazanov, Phys. Plasmas 5, 580 (1998).

${ }^{12}$ S. Lam, Phys. Fluids 8, 73 (1965).
${ }^{13}$ I. B. Bernstein and I. N. Rabinowitz, Phys. Fluids 2, 112 (1959).

${ }^{14} \mathrm{~J}$. G. Laframboise, "Theory of spherical and cylindrical langmuir probes in a collisionless, maxwellian plasma at rest," University of Toronto Institute for Aerospace Studies, UTIAS Report No. 100, 1966.

${ }^{15}$ J. R. Sanmartin and R. D. Estes, Phys. Plasmas 6, 395 (1999).

${ }^{16}$ G. Sanchez-Arriaga and J. R. Sanmartin, Phys. Plasmas 19, 063506 (2012).

${ }^{17}$ I. Langmuir and K. T. Compton, Rev. Mod. Phys. 3, 191 (1931).

${ }^{18}$ K. W. Chang and G. K. Bienkowski, Phys. Fluids 13, 902 (1970).

${ }^{19}$ X. Chen and J. R. Sanmartin, Phys. Plasmas 22, 053504 (2015).

${ }^{20}$ J. J. Schuss and R. R. Parker, J. Appl. Phys. 45, 4778 (1974).

${ }^{21}$ X. Chen and G. Sanchez-Arriaga, Phys. Plasmas 24, 023504 (2017).

${ }^{22}$ E. Choiniere, "Theory and experimental evaluation of a consistent steadystate kinetic model for 2-D conductive structures in ionospheric plasmas with application to bare electrodynamic tethers in space," Ph.D. thesis (University of Michigan, 2004).

${ }^{23}$ R. Choiniere and B. E. Gilchrist, IEEE Trans. Plasma Sci. 35, 7 (2007).

${ }^{24}$ J. R. Sanmartin, E. Choiniere, B. E. Gilchrist, J. B. Ferry, and M. Martinez-Sanchez, IEEE Trans. Plasma Sci. 36, 2851 (2008).

${ }^{25}$ G. Sanchez-Arriaga and D. Pastor-Moreno, Phys. Plasmas 21, 073504 (2014). 